\title{
Lattice dynamical systems: dissipative mechanism and fractal dimension of global and exponential attractors
}

\author{
Jan W. Cholewa And RadosŁaw CZaja(D
}

\begin{abstract}
In this work, we examine first-order lattice dynamical systems, which are discretized versions of reaction-diffusion equations on the real line. We prove the existence of a global attractor in $\ell^{2}$, and using the method by Chueshov and Lasiecka (Dynamics of quasi-stable dissipative systems, Springer, Berlin, 2015; Memoirs of the American Mathematical Society, vol 195(912), AMS, 2008), we estimate its fractal dimension. We also show that the global attractor is contained in a finite-dimensional exponential attractor. The approach relies on the interplay between the discretized diffusion and reaction, which has not been exploited as yet for the lattice systems. Of separate interest is a characterization of positive definiteness of the discretized Schrödinger operator, which refers to the well-known Arendt and Batty's result (Differ Int Equ 6:1009-1024, 1993).
\end{abstract}

\section{Introduction}

The asymptotic behavior of nonlinear reaction-diffusion equations in unbounded domains has been investigated by many authors; see, e.g., [5-7, 16,17,27,30] and references therein. A simple illustrative example is a scalar reaction-diffusion equation on the real line

$$
\begin{cases}u_{t}=\mu u_{x x}+f(x, u)+g, & x \in \mathbb{R}, \quad t>0, \\ u(0, x)=u^{0}(x), & x \in \mathbb{R},\end{cases}
$$

which can be viewed as an archetype model for the first-order lattice systems. Taking an equi-spaced discretization of (1.1) in the space variable with a step $h>0$ and letting $v:=\frac{\mu}{h^{2}}>0$ lead to the first-order system of the form

$$
\begin{cases}u_{i}^{\prime}+v\left[2 u_{i}-u_{i+1}-u_{i-1}\right]=f_{i}\left(u_{i}\right)+g_{i}, & i \in \mathbb{Z}, \quad t>0, \\ u_{i}(0)=u_{i}^{0}, & i \in \mathbb{Z},\end{cases}
$$

which will be of our interest in this paper.

Such systems, involving infinite countable number of ordinary differential equations, were broadly studied under various assumptions on the nonlinear term. On the

Mathematics Subject Classification: Primary 37L30; Secondary 37L60, 35B41, 35K57

Keywords: Lattice dynamical system, Global attractor, Fractal dimension, Reaction-diffusion equation. Jan W. Cholewa: Partially supported by the Grant MTM2016-75465 from MINECO, Spain. 
one hand, for a suitable cubic polynomial nonlinearity, traveling wave solutions were established in [36] (see also [12]). On the other hand, for (1.2) with the right-hand side of the form

$$
-\lambda u_{i}-\mathcal{F}\left(u_{i}\right)+g_{i}, \quad\left(g_{i}\right) \in \ell^{2},
$$

where

$$
\lambda>0 \text { and } \mathcal{F}(s) s \geq 0 \text { for all } s \in \mathbb{R},
$$

the strong stability properties of the system in $\ell^{2}$ were established in [8], which involved in particular the concept of a global attractor developed in [7, 20,22,29].

A similar strong stability result was proved in [33] for a generalized lattice system and in [24] for a partly dissipative system with $\lambda=\lambda_{i}$ and $\mathcal{F}=\mathcal{F}_{i}$ in (1.3) satisfying a variant of (1.4) (cf. [33, p. 53] and [24, p. 162]; also [34, p. 366]). Specifically, finite fractal dimensionality of the global attractor was shown in [35] assuming, in particular, that

$$
0<\lambda_{0} \leq \lambda_{i} \leq \lambda^{0} \text { and } \mathcal{F}_{i}(s) s \geq 0 \text { for all } s \in \mathbb{R}
$$

A nonautonomous case was considered separately in [32] with $g_{i}$ in (1.3) depending on time and $\lambda, \mathcal{F}$ as in (1.4), in which case a uniform attractor in $\ell^{2}$ was constructed for the associated family of processes. Invariant regions were additionally studied in [28].

In the further development of the theory, sufficient conditions for the existence of an exponential attractor were obtained. This was originated in [1], where such sufficient conditions were exhibited for the right-hand side (1.3) considered with $\lambda=\lambda_{i}$ and $\mathcal{F}=\mathcal{F}_{i}$ satisfying (1.5), and $\mathcal{F}_{i}$ being an odd-degree polynomial with nonnegative coefficients (see [1, p. 219]). In [19] an estimate of fractal dimension of exponential attractor for the lattice system was given, which in the similar vein involved the righthand side as in (1.3) and (1.4) above.

The above-mentioned results were obtained in the standard spaces of summable sequences, which is the setting chosen in this paper as well. However, lattice systems were also considered in weighted spaces of sequences; see, e.g., [10,21,23,31]. This is a distinct approach, since such phase spaces have different properties from the ones considered here. Specifically exponential attractors were studied in [21] and the righthand side (1.3) was considered in such setting with $\mathcal{F}=\mathcal{F}_{i}$ satisfying $\mathcal{F}_{i}(s) s \geq-b_{i}^{2}$ and $\left(g_{i}\right) \in \ell_{\rho}^{2},\left(b_{i}\right) \in \ell_{\rho}^{2}$ for some suitable weight $\rho$. We remark that therein $\lambda=\lambda_{i}$ preserved the estimate as in (1.5) (cf. [21, p. 451]).

In this paper, we will consider attracting properties of the lattice system in the strong topology of phase spaces of summable sequences assuming dissipativity conditions under which it does not fall in general into the class of problems covered by the previous results (see Example 5.4 and Remark 5.5). Namely, our approach will be based on a cooperation between the discretized diffusion and the discretized reaction. 
Hence we assume for some $\left(m_{i}\right) \in \ell^{\infty},\left(d_{i}\right) \in \ell^{2}$ the structure condition

$$
s f_{i}(s) \leq m_{i} s^{2}+d_{i}|s|, \quad i \in \mathbb{Z}, s \in \mathbb{R}
$$

requiring simultaneously that the solutions of the associated linear problem

$$
\left\{\begin{array}{l}
v_{i}^{\prime}+v\left[2 v_{i}-v_{i+1}-v_{i-1}\right]=m_{i} v_{i}, \quad t>0 \\
v_{i}(0)=v_{i}^{0}
\end{array}\right.
$$

are uniformly exponentially decaying as $t \rightarrow \infty$.

Note that this is a counterpart of a dissipative mechanism already described in a large capacity for the space continuous models (see [5,6,11]). On the other hand, observe via examples below that this essentially generalizes dissipativity assumptions used in the presented literature for proving strong stability properties of (1.2) and in particular for the existence of global and exponential attractor in $\ell^{2}$ and estimating its fractal dimension. Our main goal will be to achieve these results based on the interplay between discretized diffusion and reaction.

A brief description of the contents of the paper is as follows. In Sect. 2 we include various technicalities concerning the discretized diffusion operator and review the existence of local solutions. In Sect. 3 we construct the semigroup of global solutions following a suitable structure condition on the nonlinear term mentioned above in (1.6). In Sect. 4 the structure condition combined with the decaying properties of (1.7) described in terms of positive definiteness of the discretized Schrödinger operator will constitute a general framework for the existence of a global attractor. A discussion concerning broader applicability of such approach is given in Sect. 5. In Sect. 6, we will then estimate from above fractal dimension of the attractor using the method developed by Chueshov and Lasiecka [13,14]. Furthermore, in Sect. 7, we prove the existence of a finite dimensional exponential attractor, which contains the global attractor.

Some crucial tools used in the main body of the paper have been included in "Appendix". Of separate interest therein is a characterization of positive definiteness of the discretized Schrödinger operator, which refers to the well-known Arendt and Batty's result [4]. This allows us, in particular, to exhibit variety of sequences $\left(m_{i}\right)$ which can enter conditions (1.6) and (1.7) and thus be admissible in our approach. Namely, a nonnegative $\left(-m_{i}\right) \in \ell^{\infty}$ moves to the right spectrum of the operator given by the linear main part in (1.2) if and only if for some $C>0$ and $N \in \mathbb{N}$

$$
-\sum_{i=j-N}^{i=j+N} m_{i} \geq C \text { for all } j \in \mathbb{Z}
$$

or, equivalently, $-\sum_{i \in G} m_{i}=\infty$ for each set $G \subset \mathbb{Z}$ possessing the property that, given any $n \in \mathbb{N}$,

$$
\left\{i \in \mathbb{Z}:\left|i-j_{n}\right|<n\right\} \subset G \text { for some } j_{n} \in \mathbb{Z}
$$


(see Theorem A.3 of "Appendix"). We emphasize that for the right-hand side as in (1.3) satisfying (1.4) or (1.5) we can simply take $m_{i}=-\lambda<0$ or $m_{i}=-\lambda_{i} \leq-\lambda_{0}<0$ for which these criteria apply immediately. An example of an admissible sequence $\left(m_{i}\right)$ such that $m_{i}$ is nonzero only on some rarely distributed in $\mathbb{Z}$ subset of indices $i$ is constructed in Remark 5.2. Moreover, some sequences with infinitely many positive terms are admissible (for details, see again Remark 5.2).

Our results are, nonetheless, much more involved. Specifically Sect. 5.2 exhibits several nonlinearities for which proposed dissipative mechanism applies, which in turn generalizes previous considerations concerning global and exponential attractors for lattice dynamical systems in spaces of summable sequence. For example, following Corollary 5.8, our approach applies to

$$
f_{i}(s)=\lambda_{i} s+h_{i}(s), \quad s \in \mathbb{R}, i \in \mathbb{Z},
$$

if there is a decomposition

$$
\lambda_{i}=m_{i}+c_{i}, i \in \mathbb{Z}, \quad m, c \in \ell^{\infty}
$$

with $\left(m_{i}\right)$ as in (1.8) and

$$
h_{i}(s)=a\left(\rho_{1}\right)_{i} s|s|^{\rho_{1}-1}+\cdots+a\left(\rho_{\kappa}\right)_{i} s|s|^{\rho_{\kappa}-1}, \quad s \in \mathbb{R}, \quad i \in \mathbb{Z},
$$

where

$$
\begin{aligned}
& 1<\rho_{1}<\cdots<\rho_{\kappa}, \quad a\left(\rho_{1}\right), \ldots, a\left(\rho_{\kappa}\right) \in \ell^{\infty}, \quad a\left(\rho_{\kappa}\right)_{i}<0, i \in \mathbb{Z}, \\
& \sum_{i \in \mathbb{Z}}\left|a\left(\rho_{\kappa}\right)_{i}\right|^{-\frac{2}{\rho_{\kappa}-1}}\left|c_{i}\right|^{\frac{2 \rho_{\kappa}}{\rho_{K}-1}}<\infty,
\end{aligned}
$$

and coefficients $a\left(\rho_{j}\right)_{i}, i \in \mathbb{Z}$, with $1 \leq j<\kappa$ can have unspecified sign provided that $\sum_{i \in \mathbb{Z}}\left|a\left(\rho_{j}\right)_{i}\right|^{\frac{2 \rho_{\kappa}}{\rho_{\kappa}-\rho_{j}}}\left|a\left(\rho_{\kappa}\right)_{i}\right|^{-\frac{2 \rho_{j}}{\rho_{\kappa}-\rho_{j}}}<\infty$.

We finally remark that the main results rely on the estimates of solutions in $\ell^{2}$ and therefore the existence of attractor is established in $\ell^{2}$. Nevertheless, due to embedding properties of the scale (see (6.20)), the attractor is compact in $\ell^{p}$ for any $p \in[2, \infty]$. Also the semigroup on the attractor is Lipschitz with values in $\ell^{p}, p \in[2, \infty]$. Hence fractal dimension of the attractor is finite in $\ell^{p}$ and the estimate from above established in the Hilbert setting extends respectively (see Corollaries 6.7 and 6.10).

\section{Notation and local existence result}

We will use the Banach spaces

$$
\begin{aligned}
& \ell^{p}=\left\{u=\left(u_{i}\right): u_{i} \in \mathbb{R}, \quad i \in \mathbb{Z}, \quad \sum_{i \in \mathbb{Z}}\left|u_{i}\right|^{p}<\infty\right\}, p \in[1, \infty), \\
& \ell^{\infty}=\left\{u=\left(u_{i}\right): u_{i} \in \mathbb{R}, \quad i \in \mathbb{Z}, \quad \sup _{i \in Z}\left|u_{i}\right|<\infty\right\}
\end{aligned}
$$


with the norms $\|u\|_{p}=\left(\sum_{i \in \mathbb{Z}}\left|u_{i}\right|^{p}\right)^{\frac{1}{p}}$ and $\|u\|_{\infty}=\sup _{i \in \mathbb{Z}}\left|u_{i}\right|$, respectively. In case $p=2$ the space $\ell^{2}$ is a Hilbert space with the inner product

$$
(u, v)=\sum_{i \in \mathbb{Z}} u_{i} v_{i}, \quad u=\left(u_{i}\right), \quad v=\left(v_{i}\right) \in \ell^{2}
$$

and the norm

$$
\|u\|_{2}=\sqrt{(u, u)}=:\|u\| \text {. }
$$

We also remark that $\|u\|_{\infty} \leq\|u\|_{p}$ for $u \in \ell^{p}$ and that, given $v \in \ell^{\infty}$ and $u \in \ell^{p}$, we write $v u \in \ell^{p}$ to denote the sequence

$$
(v u)_{i}=v_{i} u_{i}, i \in \mathbb{Z} .
$$

In the $\ell^{p}$ spaces we will use isometries $\tau, \tau^{*}$,

$$
\tau, \tau^{*} \in \mathcal{L}\left(\ell^{p}\right), \quad(\tau u)_{i}=u_{i+1} \text { and }\left(\tau^{*} u\right)_{i}=u_{i-1} \quad \text { for } u=\left(u_{i}\right) \in \ell^{p}
$$

to define a linear operator

$$
A: \ell^{p} \rightarrow \ell^{p}, \quad A=\nu\left(2 \mathrm{id}-\tau-\tau^{*}\right) .
$$

Lemma 2.1. $A$ in (2.1) satisfies for every $p \in[1, \infty]$

$$
A=B B^{*}=B^{*} B,
$$

where $B, B^{*}: \ell^{p} \rightarrow \ell^{p}$ are given by

$$
B=\sqrt{v}(\tau-\mathrm{id}), \quad B^{*}=\sqrt{v}\left(\tau^{*}-\mathrm{id}\right) .
$$

Also,

$$
\|B\|_{\mathcal{L}\left(\ell^{p}\right)} \leq 2 \sqrt{\nu}, \quad\left\|B^{*}\right\|_{\mathcal{L}\left(\ell^{p}\right)} \leq 2 \sqrt{\nu}, \quad\|A\|_{\mathcal{L}\left(\ell^{p}\right)} \leq 4 v .
$$

Proof. Using $\tau \tau^{*}=\tau^{*} \tau=$ id, we get (2.2), whereas estimates (2.4) follow easily.

In what follows, we will view (1.2) in the abstract form

$$
\left\{\begin{array}{l}
u^{\prime}+A u=F(u), \quad t>0 \\
u(0)=u^{0}
\end{array}\right.
$$

where

$$
F: \ell^{p} \rightarrow \ell^{p}, \quad(F(u))_{i}=f_{i}\left(u_{i}\right)+g_{i}, i \in \mathbb{Z}, u=\left(u_{i}\right) \in \ell^{p}
$$

and

$$
g=\left(g_{i}\right) \in \ell^{p} .
$$


Observe that if

$$
\begin{aligned}
& f_{i} \in C^{1}(\mathbb{R}, \mathbb{R}) \text { is such that } f_{i}(0)=0 \text { for every } i \in \mathbb{Z} \text { and } \\
& \sup _{i \in \mathbb{Z}} \max _{|s| \leq R}\left|f_{i}^{\prime}(s)\right| \leq L_{R} \text { for every } R>0 \text { with some } L_{R} \geq 0,
\end{aligned}
$$

then the map $F$ takes $\ell^{p}$ into $\ell^{p}$ and is Lipschitz continuous on bounded sets. Indeed, given a ball $\mathcal{B}=\left\{u \in \ell^{p}:\|u\|_{p} \leq R\right\}$ and using the mean value theorem with $\theta_{i} \in[0,1]$, we have for $u, v \in \mathcal{B}$ that

$$
\|F(u)-F(v)\|_{p}^{p}=\sum_{i \in \mathbb{Z}}\left|f_{i}^{\prime}\left(\theta_{i} u_{i}+\left(1-\theta_{i}\right) v_{i}\right)\right|^{p}\left|u_{i}-v_{i}\right|^{p} \leq L_{R}^{p}\|u-v\|_{p}^{p},
$$

because $\left|\theta_{i} u_{i}+\left(1-\theta_{i}\right) v_{i}\right| \leq \theta_{i}\|u\|_{p}+\left(1-\theta_{i}\right)\|v\|_{p} \leq R$.

Due to the properties of $A$ and $F$, the general theory of abstract differential equations leads to the existence result as stated below.

Proposition 2.2. Given $p \in[1, \infty]$ we assume (2.6) and (2.7). Then for any $u^{0} \in$ $\ell^{p}$ there exists a unique solution $u \in C^{1}\left(\left[0, t_{\max }\left(u^{0}\right)\right), \ell^{p}\right)$ of $(2.5)$ defined on the maximal interval of existence $\left[0, t_{\max }\left(u^{0}\right)\right)$ and $\lim _{t \rightarrow t_{\max }\left(u^{0}\right)}\left\|u\left(t ; u^{0}\right)\right\|_{p}=\infty$ unless $t_{\max }\left(u^{0}\right)=\infty$.

Remark 2.3. Besides the $\ell^{p}$ setting as above, in the following sections, we will rely on the estimates of the solution in $\ell^{2}$. Hence we observe that $A: \ell^{2} \rightarrow \ell^{2}$ is a self-adjoint nonnegative definite operator with spectrum in $[0, \infty)$. Indeed, since

$$
\begin{aligned}
(B u, v) & =\sqrt{v}(\tau u-u, v)=\sqrt{v} \sum_{i \in \mathbb{Z}}\left(u_{i+1} v_{i}-u_{i} v_{i}\right) \\
& =\sqrt{v} \sum_{i \in \mathbb{Z}} u_{i}\left(v_{i-1}-v_{i}\right)=\sqrt{v}\left(u, \tau^{*} v-v\right)=\left(u, B^{*} v\right), u, v \in \ell^{2},
\end{aligned}
$$

using this and (2.2) we get

$$
(A u, u)=\left(B B^{*} u, u\right)=\left\|B^{*} u\right\|^{2}=\|B u\|^{2} \geq 0, u \in \ell^{2},
$$

and we also obtain in a similar manner that $(A u, v)=(u, A v), u, v \in \ell^{2}$.

\section{Global existence}

We now restrict our considerations to the Hilbert case $p=2$. Therefore, we assume that the nonlinearity satisfies (2.7) and

$$
g=\left(g_{i}\right) \in \ell^{2} .
$$


The aim of the section is to show that the solutions in $\ell^{2}$ exist globally in time, i.e., $t_{\max }\left(u^{0}\right)=\infty$ for any $u^{0} \in \ell^{2}$. To this end, we introduce the structure condition for the nonlinearity

$$
\begin{aligned}
& s f_{i}(s) \leq m_{i} s^{2}+d_{i}|s|, i \in \mathbb{Z}, s \in \mathbb{R} \\
& \text { for some } m=\left(m_{i}\right) \in \ell^{\infty}, d=\left(d_{i}\right) \in \ell^{2} .
\end{aligned}
$$

Note that it is implicit in (3.2) that $d_{i} \geq 0$ for $i \in \mathbb{Z}$.

The global existence of solutions of (2.5) is a consequence of the following lemma.

Lemma 3.1. For any $u^{0} \in \ell^{2}$, there exists a constant $C=C\left(\left\|u^{0}\right\|\right) \geq 0$ such that

$$
\left\|u\left(t ; u^{0}\right)\right\| \leq C e^{\left(\|m\|_{\infty}+1\right) t}, t \in\left[0, t_{\max }\left(u_{0}\right)\right) .
$$

Hence we obtain $t_{\max }\left(u_{0}\right)=\infty$.

Proof. We take the inner product of the first equation in (2.5) with $u$ in $\ell^{2}$ and get

$$
\frac{1}{2} \frac{\mathrm{d}}{\mathrm{d} t}\|u\|^{2}+(A u, u)=(F(u), u) .
$$

Since

$$
(F(u), u) \leq \sum_{i \in \mathbb{Z}}\left(m_{i} u_{i}^{2}+d_{i}\left|u_{i}\right|+g_{i} u_{i}\right) \leq\left(\|m\|_{\infty}+1\right)\|u\|^{2}+\frac{\|d\|^{2}+\|g\|^{2}}{2},
$$

we obtain by the nonnegativity of $A$

$$
\frac{\mathrm{d}}{\mathrm{d} t}\|u\|^{2} \leq 2\left(\|m\|_{\infty}+1\right)\|u\|^{2}+\|d\|^{2}+\|g\|^{2} .
$$

By the Gronwall inequality, we get (3.3).

Therefore we define on $\ell^{2}$ the semigroup $\{S(t): t \geq 0\}$ of global solutions of (2.5) by

$$
S(t) u^{0}=u\left(t ; u^{0}\right), t \geq 0, u^{0} \in \ell^{2} .
$$

This is a $C^{0}$ semigroup in the sense that

$$
[0, \infty) \times \ell^{2} \ni\left(t, u^{0}\right) \mapsto S(t) u^{0} \in \ell^{2} \text { is continuous. }
$$

Remark 3.2. Note that if maps in (2.7) satisfy

$$
f_{i}^{\prime}(s) \leq m_{i}, s \in \mathbb{R}, i \in \mathbb{Z} \text {, with }\left(m_{i}\right) \in \ell^{\infty},
$$

then (3.2) holds with $d_{i}=0$ for $i \in \mathbb{Z}$, but the condition (3.2) is much more flexible than (3.6) (see Remarks 5.6, 5.7). 


\section{Dissipativity and global attractor}

In this section for the semigroup of global solutions in $\ell^{2}$, we prove the existence of a bounded absorbing set and establish asymptotic compactness. As mentioned in the introduction our approach will be based on a cooperation between the discretized diffusion and the discretized reaction terms. To this end, simultaneously with (3.2), we require for the linear problem

$$
\left\{\begin{array}{l}
v^{\prime}+A v=m v, \quad t>0, \\
v(0)=v^{0}
\end{array}\right.
$$

that the linear semigroup $e^{(-A+m) t}$ on $\ell^{2}$ generated by $-A+m$ is uniformly exponentially stable ([18, Definition I.3.11]). This is equivalent to the requirement that the spectrum of the self-adjoint operator $A-m$ in $\ell^{2}$ is positive

$$
\inf \left\{\lambda: \lambda \in \sigma_{2}(A-m)\right\}>0 .
$$

This also means that the operator $A-m$ is positive definite, i.e., there exists a positive constant $\alpha_{0}>0$ such that

$$
(A u, u)-(m u, u) \geq \alpha_{0}\|u\|^{2}, u \in \ell^{2} .
$$

To find suitable estimates of solutions observe that with the above-mentioned assumptions, the nonlinear term after taking a product with $u$ cannot be dropped, which would be the case for the right-hand side as in (1.3) and (1.4). Differently than in Sect. 3 the linear main part after taking a product with $u$ cannot be dropped either. Actually, both the discretized diffusion and discretized reaction must intervene to produce desired dissipativity and asymptotic compactness effects.

Theorem 4.1. Assume (2.7), (3.1), (3.2) and (4.2). Given any $M>0$ satisfying

$$
M>\frac{\|d\|+\|g\|}{\alpha_{0}},
$$

for each bounded subset $\mathcal{B}$ of $\ell^{2}$ there exists $T_{\mathcal{B}} \geq 0$ such that

$$
\forall_{t \geq T_{\mathcal{B}}} \forall_{u^{0} \in \mathcal{B}}\left\|u\left(t ; u^{0}\right)\right\| \leq M .
$$

Moreover, if $u^{0} \in \mathcal{B}_{0}=\left\{u \in \ell^{2}:\|u\| \leq M\right\}$, then $u\left(t ; u^{0}\right) \in \mathcal{B}_{0}$ for all $t \geq 0$.

Proof. We return to (3.4) and get

$$
\frac{1}{2} \frac{\mathrm{d}}{\mathrm{d} t}\|u\|^{2}+(A u, u)-(m u, u) \leq(\|d\|+\|g\|)\|u\| .
$$

By assumption (4.2) and the Cauchy inequality, we get

$$
\frac{\mathrm{d}}{\mathrm{d} t}\|u\|^{2}+\alpha_{0}\|u\|^{2} \leq \frac{1}{\alpha_{0}}(\|d\|+\|g\|)^{2}
$$

and by the Gronwall inequality we get the result. 
Now we use the idea of [8, Lemma 2.3] (cf. also [24]) to estimate the tail ends of solutions. Since our assumptions are different from the ones used in the mentioned references we proceed with the direct proofs of the two lemmas below. Then standard argument will ensure the asymptotic compactness used in Theorem 4.4.

Let $\theta:[0, \infty) \rightarrow[0,1]$ be a $C^{1}$ function $^{1}$ such that

$$
\theta(s)= \begin{cases}0, & 0 \leq s \leq 1 \\ 1, & s \geq 2\end{cases}
$$

and $\left|\theta^{\prime}(s)\right| \leq 2$ for $s \geq 0$. We fix $k \in \mathbb{N}$ and consider a sequence $\eta_{k} \in \ell^{\infty}$ given by $\left(\eta_{k}\right)_{i}=\theta\left(\frac{|i|}{k}\right), i \in \mathbb{Z}$.

Lemma 4.2. For any $u \in \ell^{2}$, we have

$$
\left(B u, B\left(\eta_{k}^{2} u\right)\right)=\left\|B\left(\eta_{k} u\right)\right\|^{2}+R(k, u),
$$

where $B$ is as in Lemma 2.1 and

$$
|R(k, u)| \leq \frac{20 v}{k}\|u\|^{2} .
$$

Proof. Using the formula

$$
B(\eta u)=(B \eta) \tau u+\eta B u, \quad \eta \in \ell^{\infty}, \quad u \in \ell^{2},
$$

we get

$$
\left(B u, B\left(\eta_{k}^{2} u\right)\right)=\left\|\eta_{k} B u\right\|^{2}+\left(B u,\left(B \eta_{k}^{2}\right) \tau u\right)
$$

and

$$
\left(B\left(\eta_{k} u\right), B\left(\eta_{k} u\right)\right)=\left\|\eta_{k} B u\right\|^{2}+2\left(\eta_{k} B u,\left(B \eta_{k}\right) \tau u\right)+\left\|\left(B \eta_{k}\right) \tau u\right\|^{2} .
$$

Thus

$\left(B u, B\left(\eta_{k}^{2} u\right)\right)=\left\|B\left(\eta_{k} u\right)\right\|^{2}-2\left(\eta_{k} B u,\left(B \eta_{k}\right) \tau u\right)-\left\|\left(B \eta_{k}\right) \tau u\right\|^{2}+\left(B u,\left(B \eta_{k}^{2}\right) \tau u\right)$.

Note that

$$
\left\|B \eta_{k}\right\|_{\infty}=\sqrt{v} \sup _{i \in \mathbb{Z}}\left|\theta\left(\frac{|i+1|}{k}\right)-\theta\left(\frac{|i|}{k}\right)\right| \leq \frac{2 \sqrt{v}}{k} .
$$

We have by the Cauchy-Schwarz inequality

$$
\begin{aligned}
& 2\left|\left(\eta_{k} B u,\left(B \eta_{k}\right) \tau u\right)\right| \leq 2\left\|\eta_{k} B u\right\|\left\|\left(B \eta_{k}\right) \tau u\right\| \leq \frac{8 v}{k}\|u\|^{2}, \\
& \left\|\left(B \eta_{k}\right) \tau u\right\|^{2} \leq\left\|B \eta_{k}\right\|_{\infty}^{2}\|\tau u\|^{2} \leq \frac{4 v}{k^{2}}\|u\|^{2}, \\
& \left|\left(B u,\left(B \eta_{k}^{2}\right) \tau u\right)\right| \leq\|B u\|\left\|B \eta_{k}^{2}\right\|_{\infty}\|\tau u\| \leq \frac{8 v}{k}\|u\|^{2},
\end{aligned}
$$

which ends the proof.

${ }^{1}$ For example, we set $\theta(s)=\frac{\varphi(s-1)}{\varphi(s-1)+\varphi(2-s)}, s \geq 0$, where $\varphi(s)=e^{-\frac{1}{s}}$ if $s>0$ and $\varphi(s)=0$ if $s \leq 0$. 
Lemma 4.3. Suppose that the assumptions of Theorem 4.1 hold. Given $\varepsilon>0$ there exist $k_{\varepsilon} \in \mathbb{N}$ and $T_{\varepsilon}>0$ such that for any $t \geq T_{\varepsilon}$ and any $u^{0}$ in a bounded positively invariant absorbing set $\mathcal{B}_{0}$ from Theorem 4.1 , we have

$$
\sum_{|i|>k_{\varepsilon}}\left|u_{i}\left(t ; u^{0}\right)\right|^{2}<\varepsilon
$$

Proof. Taking the inner product of the first equation in (2.5) with $\eta_{k}^{2} u$ in $\ell^{2}$, we get

$$
\frac{1}{2} \frac{\mathrm{d}}{\mathrm{d} t}\left\|\eta_{k} u\right\|^{2}+\left(A u, \eta_{k}^{2} u\right)=\left(F(u), \eta_{k}^{2} u\right)
$$

Note that by (2.9) and Lemma 4.2

$$
\left(A u, \eta_{k}^{2} u\right)=\left(B u, B\left(\eta_{k}^{2} u\right)\right) \geq\left(A\left(\eta_{k} u\right), \eta_{k} u\right)-\frac{20 v}{k}\|u\|^{2},
$$

whereas by the structure condition (3.2) and the fact that $\eta_{k}^{2} \leq \eta_{k}$, we get

$$
\left(F(u), \eta_{k}^{2} u\right) \leq\left(m\left(\eta_{k} u\right), \eta_{k} u\right)+\sum_{|i|>k}\left(d_{i}+\left|g_{i}\right|\right)\left(\eta_{k}\right)_{i}\left|u_{i}\right| .
$$

Since (4.2) holds with $\alpha_{0}>0$, using in the last term the Cauchy inequality with $\alpha_{0}$, we obtain

$$
\left(F(u), \eta_{k}^{2} u\right) \leq\left(m\left(\eta_{k} u\right), \eta_{k} u\right)+\frac{\alpha_{0}}{2}\left\|\eta_{k} u\right\|^{2}+\frac{1}{2 \alpha_{0}} \sum_{|i|>k}\left(d_{i}+\left|g_{i}\right|\right)^{2} .
$$

Combining (4.5), (4.6) and the assumption (4.2) with (4.4), we obtain for $k \in \mathbb{N}$

$$
\frac{d}{d t}\left\|\eta_{k} u\right\|^{2}+\alpha_{0}\left\|\eta_{k} u\right\|^{2} \leq \frac{40 v}{k}\|u\|^{2}+\frac{1}{\alpha_{0}} \sum_{|i|>k}\left(d_{i}+\left|g_{i}\right|\right)^{2} .
$$

Fix $\varepsilon>0$ and let $I_{\varepsilon} \in \mathbb{N}$ be such that

$$
\frac{40 v M^{2}}{I_{\varepsilon}}+\frac{1}{\alpha_{0}} \sum_{|i|>I_{\varepsilon}}\left(d_{i}+\left|g_{i}\right|\right)^{2} \leq \frac{\alpha_{0} \varepsilon}{2},
$$

where $M>0$ is as in Theorem 4.1. For any $t>0$ and $u^{0} \in \mathcal{B}_{0}$, we have $u\left(t ; u^{0}\right) \in \mathcal{B}_{0}$ and

$$
\frac{\mathrm{d}}{\mathrm{d} t}\left\|\eta_{I_{\varepsilon}} u\right\|^{2}+\alpha_{0}\left\|\eta_{I_{\varepsilon}} u\right\|^{2} \leq \frac{\alpha_{0} \varepsilon}{2} .
$$

Applying the Gronwall inequality, we get the claim with $k_{\varepsilon}=2 I_{\varepsilon}$.

Theorem 4.4. Under the assumptions (2.7), (3.1), (3.2) and (4.2), the system (1.2) generates a semigroup $\{S(t): t \geq 0\}$ on $\ell^{2}$ possessing a global attractor $\mathcal{A}$, that is, a nonempty compact subset of $\ell^{2}$, invariant under the semigroup, and such that for any bounded subset $\mathcal{B}$ of $\ell^{2}$

$$
d(S(t) \mathcal{B}, \mathcal{A})=\sup _{u \in \mathcal{B}} \inf _{v \in \mathcal{A}}\|S(t) u-v\| \rightarrow 0 \text { as } t \rightarrow \infty .
$$


Proof. Since by Theorem 4.1 the semigroup is bounded dissipative, it suffices to show that it is asymptotically compact (see [22]). With Theorem 4.1 and Lemma 4.3, the proof of asymptotic compactness is standard, and hence, it will be omitted (for details see, e.g., [8, pp. 147-148] or [24, proof of Lemma 3.3]).

\section{Remarks concerning dissipative mechanism}

In this section, we make a few remarks on the assumptions (3.2) and (4.2) which are at the core of the dissipative mechanism.

\subsection{Bottom spectrum of discretized Schrödinger operator}

Recall that the spectrum of the operator

$$
A u=v\left(2 u-\tau u-\tau^{*} u\right), \quad u \in \ell^{2},
$$

is contained in $[0, \infty)$ (see Remark 2.3) and observe that if, for example, the sequence $m$ is a negative constant as in $[8,35]$, that is,

$$
m_{i}=-\alpha_{0}<0, \quad i \in \mathbb{Z},
$$

then (4.2) is satisfied and Theorem 4.4 applies.

To show a broader applicability of Theorem 4.4, it is crucial to give a characterization of the sequences which move the spectrum of the operator $A$ to the right. The result below is a consequence of Theorem A.3 proved in "Appendix" by modifying Arendt and Batty's [4, Theorem 1.2].

Proposition 5.1. A necessary and sufficient condition for $0 \leq-m \in \ell^{\infty}$ to satisfy (4.1) is that $-\sum_{i \in G} m_{i}=\infty$ for each set $G \subset \mathbb{Z}$ possessing the property that, given any $n \in \mathbb{N}$,

$$
\left\{i \in \mathbb{Z}:\left|i-j_{n}\right|<n\right\} \subset G \text { for some } j_{n} \in \mathbb{Z} \text {. }
$$

Hence observe that in (3.2) and (4.1), we can actually have sequences $m \in \ell^{\infty}$ with infinitely many positive terms (see Remark 5.2). Consequently, our argument for the existence of the global attractor for (1.2) essentially generalizes [8,35] (see Corollary 5.3, Remarks 5.6, 5.7 and Corollary 5.8).

Remark 5.2. (i) One can construct a nonconstant $0 \leq-m \in \ell^{\infty}$ satisfying the condition in Proposition 5.1. Indeed, for a fixed $r>0$ and $n \in \mathbb{N}$, choose an integer $0 \leq k<n$ and define

$$
\left(\widetilde{m}_{r, k, n}\right)_{i}= \begin{cases}-r, & i=0, \ldots, k \\ 0, & i=k+1, \ldots n-1 .\end{cases}
$$

Denote by $m=m_{r, k, n} \in \ell^{\infty}$ the periodic extension of $\tilde{m}_{r, k, n}$ to $\mathbb{Z}$. Then, given $G \in \mathcal{G}$ as in Definition A.1 and an integer $p \geq 1$, the interval $\left(j_{p n}-p n, j_{p n}+p n\right)$ contains 
$2 p-1$ intervals of length $n$ having the form $[\ln , \ln +n)$ for some integer $l$ and, since $m_{i}=-r$ for $i \in[\ln , \ln +k+1) \subset[\ln , \ln +n)$,

$$
-\sum_{i \in G} m_{i} \geq-\sum_{i \in\left(j_{p n}-p n, j_{p n}+p n\right) \cap \mathbb{Z}} m_{i} \geq(2 p-1) r(k+1) .
$$

Observe that, due to Proposition 5.1, $m=m_{r, k, n}$ satisfies (4.1) and thus also (4.2) with some $\alpha_{0}>0$. Furthermore, number of zeros between nonzero components of $m=m_{r, k, n}$ equals $n-k-1$, and therefore, it can be as large as we intend.

The property that $-\sum_{i \in G} m_{i}=\infty$ for any $G \in \mathcal{G}$, and hence (4.1), will also hold for $m=\chi_{N} m_{r, k, n}$, where

$$
\left(\chi_{N}\right)_{i}= \begin{cases}0, & |i| \leq N \\ 1, & |i|>N\end{cases}
$$

and $N$ is an arbitrarily large positive integer.

(ii) Given $m \in \ell^{\infty}$ and $\alpha_{0}>0$ as in (4.2) observe further that if $\hat{m} \in \ell^{\infty}$ is any sequence with $\|\hat{m}\|_{\infty}<\alpha_{0}$, then $(A u, u)-((m+\hat{m}) u, u) \geq\left(\alpha_{0}-\|\hat{m}\|_{\infty}\right)\|u\|^{2}$ for all $u \in \ell^{2}$. In particular, if zero components of the sequence $m$ from (i), which satisfies (4.1), are replaced by a positive number less than $\alpha_{0}$, then the resulting sequence will still maintain (4.1).

\subsection{Sample nonlinearities}

Let us now make a few remarks concerning nonlinearities for which a general mechanism described in the previous section applies. To proceed observe that $C^{1}$ maps $f_{i}: \mathbb{R} \rightarrow \mathbb{R}, i \in \mathbb{Z}$, with

$$
f_{i}(0)=0, \quad i \in \mathbb{Z},
$$

can be written as

$$
f_{i}(s)=\lambda_{i} s+h_{i}(s), \quad s \in \mathbb{R}, \quad i \in \mathbb{Z}
$$

for some $\lambda_{i} \in \mathbb{R}, i \in \mathbb{Z}$, and functions $h_{i}: \mathbb{R} \rightarrow \mathbb{R}$ of the class $C^{1}$ satisfying $h_{i}(0)=0$.

A simple application of Theorem 4.4 is given in the corollary below in a situation when $\left(\lambda_{i}\right)$ moves spectrum of $A$ to the right.

Corollary 5.3. If

$$
\lambda_{i}=m_{i}, i \in \mathbb{Z},
$$

with $m=\left(m_{i}\right) \in \ell^{\infty}$ as in (4.2), $g=\left(g_{i}\right) \in \ell^{2}$, and if $h_{i}$ from (5.2) satisfy

$$
\operatorname{sh}_{i}(s) \leq d_{i}|s| \text { for } s \in \mathbb{R}, i \in \mathbb{Z}, d \in \ell^{2},
$$

and

$$
h_{i}(0)=0, i \in \mathbb{Z} \text { and } \sup _{i \in \mathbb{Z}} \max _{|s| \leq R}\left|h_{i}^{\prime}(s)\right| \leq \tilde{L}_{R} \text { for every } R>0 \text { with some } \tilde{L}_{R} \geq 0,
$$

then the semigroup $\{S(t): t \geq 0\}$ on $\ell^{2}$ generated by (1.2) has a global attractor $\mathcal{A}$. 
Proof. All assumptions of Theorem 4.4, including the structure and spectral conditions (3.2) and (4.2), are satisfied.

Example 5.4. We present an illustrative example of a lattice dynamical system, which is a special case of problems considered in Corollary 5.3.

For arbitrarily chosen $r>0, n \in \mathbb{N}$ and $N \in \mathbb{N}$ let $\bar{m}=\chi_{N} m_{r, 0, n} \in \ell^{\infty}$ be the sequence defined in Remark 5.2 (i). Therefore, letting

$$
Z_{n N}=\{i \in \mathbb{Z}:|i| \leq N \text { or }|i|>N \text { and } i \neq j n \text { for all } j \in \mathbb{Z}\}
$$

we see that

$$
\bar{m}_{i}= \begin{cases}0, & i \in Z_{n N} \\ -r, & i \notin Z_{n N}\end{cases}
$$

and by Proposition 5.1 there exists $\alpha>0$ such that

$$
(A u, u)-(\bar{m} u, u) \geq \alpha\|u\|^{2}, u \in \ell^{2} .
$$

Let

$$
\widehat{m}_{i}= \begin{cases}\frac{\alpha}{2}, & i \in Z_{n N} \\ 0, & i \notin Z_{n N}\end{cases}
$$

and set $m=\bar{m}+\widehat{m}$; hence $m=\left(m_{i}\right) \in \ell^{\infty}$ is given by

$$
m_{i}= \begin{cases}\frac{\alpha}{2}, & i \in Z_{n N} \\ -r, & i \notin Z_{n N}\end{cases}
$$

and we have (4.2) for $\alpha_{0}=\frac{\alpha}{2}>0$, that is,

$$
(A u, u)-(m u, u) \geq \alpha_{0}\|u\|^{2}, \quad u \in \ell^{2} .
$$

We emphasize that the sequence $m=\left(m_{i}\right)$ has positive values except for the indices $i$ being integer multiples of $n$, which have modulus larger than $N$.

Now we fix arbitrary sequences $a=\left(a_{i}\right) \in \ell^{6}$ and $g=\left(g_{i}\right) \in \ell^{2}$ and define

$$
h_{i}(s)= \begin{cases}0, & i \in Z_{n N}, s \in \mathbb{R}, \\ a_{i} s|s|-s^{3}, & i \notin Z_{n N}, s \in \mathbb{R},\end{cases}
$$

to consider the corresponding nonlinear system

$$
\left\{\begin{array}{l}
u_{i}^{\prime}+v\left[2 u_{i}-u_{i+1}-u_{i-1}\right]=m_{i} u_{i}+h_{i}\left(u_{i}\right)+g_{i}, \quad i \in \mathbb{Z}, t>0, \\
u_{i}(0)=u_{i}^{0}, \quad i \in \mathbb{Z}
\end{array}\right.
$$


Then the assumptions of Corollary 5.3 are satisfied with $\lambda_{i}=m_{i}$ given in (5.3), $h$ as in (5.4) and $g$ fixed above. Note that condition $s h_{i}(s) \leq d_{i}|s|$ therein is satisfied for $s \in \mathbb{R}$ with $d=\left(d_{i}\right) \in \ell^{2}$ given by

$$
d_{i}= \begin{cases}0, & i \in Z_{n N}, \\ \frac{1}{3}\left|a_{i}\right|^{3}, & i \notin Z_{n N},\end{cases}
$$

because using Young's inequality, we get

$$
a_{i}|s|^{3}-s^{4}=a_{i}|s|^{\frac{1}{3}}|s|^{\frac{8}{3}}-s^{4} \leq \frac{1}{3}\left|a_{i}\right|^{3}|s|+\frac{2}{3}|s|^{4}-s^{4} \leq \frac{1}{3}\left|a_{i}\right|^{3}|s|, \quad s \in \mathbb{R} .
$$

Thus (5.5) generates a semigroup $\{S(t): t \geq 0\}$ on $\ell^{2}$, which has a global attractor $\mathcal{A}$ in $\ell^{2}$.

Remark 5.5. Concerning Example 5.4 observe that it essentially enlarges admissible in the references right-hand sides. In particular, (5.5) cannot be handled as a problem with a right-hand side of the form $-\lambda_{i} u_{i}-\mathcal{F}_{i}\left(u_{i}\right)+g_{i}^{0}$ satisfying (1.5); nor even satisfying $\lambda_{i}>0$ and $\mathcal{F}_{i}(s) s \geq-b_{i}^{2}$ for all $i \in \mathbb{Z}$ and $s \in \mathbb{R}$ (see [21, Section 3.1]). Indeed, supposing the latter was true one would have

$$
m_{i} s+h_{i}(s)+g_{i}=-\lambda_{i} s-\mathcal{F}_{i}(s)+g_{i}^{0}, s \in \mathbb{R}, i \in \mathbb{Z} .
$$

For $i \in Z_{n N}$ this would yield by (5.3), (5.4) that

$$
\frac{\alpha}{2} s^{2}+g_{i} s \leq-\lambda_{i} s^{2}+b_{i}^{2}+g_{i}^{0} s, s \in \mathbb{R},
$$

which is absurd for large $|s|$.

We now remark that our approach also applies to nonlinearities (5.2) with sequences $\left(\lambda_{i}\right)$, which may not shift the spectrum of the operator $A$ to the right.

Remark 5.6. (i) If $h_{i}$ from (5.2) satisfies

$s h_{i}(s) \leq-b_{i}|s|^{\rho+1}+\hat{d}_{i}|s|$ for $s \in \mathbb{R}, i \in \mathbb{Z}$ with some $\rho>1, b_{i}>0, \hat{d} \in \ell^{2}$,

and if there is a decomposition

$$
\lambda_{i}=m_{i}+c_{i}, i \in \mathbb{Z}, \quad m, c \in \ell^{\infty}
$$

with $m$ as in (4.1), then (3.2) will be satisfied with $d_{i}=\hat{d}_{i}+b_{i}^{-\frac{1}{\rho-1}}\left|c_{i}\right|^{\frac{\rho}{\rho-1}}, i \in \mathbb{Z}$, provided that

$$
\sum_{i \in \mathbb{Z}} b_{i}^{-\frac{2}{\rho-1}}\left|c_{i}\right|^{\frac{2 \rho}{\rho-1}}<\infty
$$

as a consequence of Young's inequality. 
(ii) Hence $c$ in part (i) above not only is allowed to have components $c_{i}$ with oscillating sign but also, taking into consideration the convergence of the series in (5.8), the sequence $c$ can have modes $c_{i}$ with $|i| \leq N$ as large as we intend to choose. Consequently, we can handle nonlinearities $f_{i}$ satisfying (5.1)-(5.7) with any values of $\lambda_{i},|i| \leq N$, for any fixed $N \in \mathbb{N}$.

Remark 5.7. Continuously differentiable maps $h_{i}: \mathbb{R} \rightarrow \mathbb{R}$ satisfying (5.6) are, for example, of the form

$$
h_{i}(s)=a\left(\rho_{1}\right)_{i} s|s|^{\rho_{1}-1}+\ldots+a\left(\rho_{\kappa}\right)_{i} s|s|^{\rho_{\kappa}-1}, s \in \mathbb{R}, i \in \mathbb{Z},
$$

where

$$
1<\rho_{1}<\cdots<\rho_{\kappa}, \quad a\left(\rho_{1}\right), \ldots, a\left(\rho_{\kappa}\right) \in \ell^{\infty}, \quad a\left(\rho_{\kappa}\right)_{i}<0, i \in \mathbb{Z},
$$

and coefficients $a\left(\rho_{j}\right)_{i}, i \in \mathbb{Z}$, with $1 \leq j<\kappa$ can have unspecified sign provided that

$$
\sum_{i \in \mathbb{Z}}\left|a\left(\rho_{j}\right)_{i}\right|^{\frac{2 \rho_{\kappa}}{\rho_{K}-\rho_{j}}}\left|a\left(\rho_{\kappa}\right)_{i}\right|^{-\frac{2 \rho_{j}}{\rho_{\kappa}-\rho_{j}}}<\infty
$$

[(5.11) not needed if $\kappa=1]$. Then (5.6) holds with $\rho=\rho_{\kappa}$ and

$$
b_{i}=-(1-\varepsilon) a\left(\rho_{\kappa}\right)_{i}, i \in \mathbb{Z} \text { for a fixed } \varepsilon \in(0,1)
$$

(and $\varepsilon=0$ if $\kappa=1$ ). Joining (5.1), (5.2), (5.7) and (5.9), we also see that (2.7) holds with

$$
L_{R}=\|m+c\|_{\infty}+\sum_{j=1}^{\kappa} \rho_{j}\left\|a\left(\rho_{j}\right)\right\|_{\infty} R^{\rho_{j}-1}, \quad R>0 .
$$

The following corollary, which builds upon Remarks 5.6 and 5.7, shows a broader applicability of Theorem 4.4.

Corollary 5.8. Let $g=\left(g_{i}\right) \in \ell^{2}$ and suppose that nonlinearities are of the form $f_{i}(s)=\lambda_{i} s+h_{i}(s), s \in \mathbb{R}, i \in \mathbb{Z}$, where $h_{i}$ are given by (5.9), (5.10), and (5.11). Assume also that $\lambda_{i}=m_{i}+c_{i}, i \in \mathbb{Z}$, with $m=\left(m_{i}\right) \in \ell^{\infty}$ and $c=\left(c_{i}\right) \in \ell^{\infty}$ such that (4.1) holds and

$$
\sum_{i \in \mathbb{Z}}\left|a\left(\rho_{\kappa}\right)_{i}\right|^{-\frac{2}{\rho_{\kappa}-1}}\left|c_{i}\right|^{\frac{2 \rho_{\kappa}}{\rho_{\kappa}-1}}<\infty .
$$

Then the semigroup $\{S(t): t \geq 0\}$ generated by (1.2) on $\ell^{2}$ possesses a global attractor $\mathcal{A}$. 


\section{Estimate of fractal dimension of attractor}

Assuming additionally to (2.7) and (3.2) that

$$
\sup _{i \in \mathbb{Z}}\left|f_{i}^{\prime}(s)-f_{i}^{\prime}(0)\right| \rightarrow 0 \text { as } s \rightarrow 0,
$$

we will now prove that the attractor in Theorem 4.4 has finite fractal dimension.

Remark 6.1. (i) Assumption (6.1) typically holds if

$$
f_{i} \in C^{2}(\mathbb{R}, \mathbb{R}) \text { for } i \in \mathbb{Z} \text { and } \sup _{i \in \mathbb{Z}} \max _{|s| \leq R}\left|f_{i}^{\prime \prime}(s)\right| \leq C_{R} \text { for } R>0 .
$$

(ii) For nonlinearities of the form (5.2), the condition (6.1) reads

$$
\sup _{i \in \mathbb{Z}}\left|h_{i}^{\prime}(s)-h_{i}^{\prime}(0)\right| \rightarrow 0 \text { as } s \rightarrow 0
$$

and holds, in particular, for $h_{i}$ given in (5.9).

Theorem 6.2. Besides the assumptions of Theorem 4.4, that is (2.7), (3.1), (3.2) and (4.2), assume also (6.1). Then the fractal dimension of the global attractor $\mathcal{A}$ in Theorem 4.4 is finite and its estimate from above is given by (6.4) and (6.19).

The proof of Theorem 6.2 will follow in a sequence of lemmas. This includes, in particular, the estimate of $\operatorname{dim}_{f}(\mathcal{A})$ in $\ell^{2}$ given in (6.4) and (6.19) (or (6.10)).

Lemma 6.3. Under the assumptions of Theorem 4.4, let $\{S(t): t \geq 0\}$ be the $C^{0}$ semigroup in $\ell^{2}$ (see (3.5)) with the global attractor $\mathcal{A}$ considered therein. If, given any $u^{0}, v^{0} \in \mathcal{A}$, inequalities

$$
\sup _{t \in\left[T_{1}, T_{2}\right]}\left\|S(t) u^{0}-S(t) v^{0}\right\|+\sup _{t \in\left[T_{1}, T_{2}\right]}\left\|\frac{d}{d t}\left(S(t) u^{0}-S(t) v^{0}\right)\right\| \leq \kappa\left\|u^{0}-v^{0}\right\|,
$$

and

$$
\left\|S\left(T_{2}\right) u^{0}-S\left(T_{2}\right) v^{0}\right\| \leq \eta\left\|u^{0}-v^{0}\right\|+\left(D \int_{T_{1}}^{T_{2}} \sum_{|i| \leq k}\left(S(s) u^{0}-S(s) v^{0}\right)_{i}^{2} d s\right)^{\frac{1}{2}}
$$

hold with some constants $\kappa>0, \eta \in[0,1), k \in \mathbb{N}, T_{2}>T_{1} \geq 0$ and $D>0$, then the fractal dimension of $\mathcal{A}$ in $\ell^{2}$ is finite. Actually, setting $Z=C^{1}\left(\left[T_{1}, T_{2}\right] ; \ell^{2}\right)$ with the norm $\|z\|_{Z}=\sup _{t \in\left[T_{1}, T_{2}\right]}\|z(t)\|+\sup _{t \in\left[T_{1}, T_{2}\right]}\left\|z^{\prime}(t)\right\|$, we have

$$
\operatorname{dim}_{f}(\mathcal{A}) \leq \frac{\ln \mathfrak{m}_{\mathcal{B}_{\eta}}}{\ln \frac{2}{1+\eta}}
$$


where $\mathfrak{m}_{\mathcal{B}_{\eta}}$ is the maximal number of $z^{j}$ in $\mathcal{B}_{\eta}:=\left\{z \in Z:\|z\|_{Z} \leq \frac{4 D^{\frac{1}{2}} \kappa}{1-\eta}\right\}$ possessing the property that

$$
\int_{T_{1}}^{T_{2}} \sum_{|i| \leq k}\left(z^{j}(t)-z^{l}(t)\right)_{i}^{2} d t>1 \text { whenever } j \neq l .
$$

Proof. Note that by the Arzela-Ascoli theorem, the seminorm

$$
n_{Z}(z)=\left(D \int_{T_{1}}^{T_{2}} \sum_{|i| \leq k}(z(t))_{i}^{2} \mathrm{~d} t\right)^{\frac{1}{2}}
$$

is compact on $Z=C^{1}\left(\left[T_{1}, T_{2}\right] ; \ell^{2}\right)$. Thus we apply Proposition B.1 specifying therein $V=S\left(T_{2}\right), \mathbb{M}=\mathcal{A}, X=\ell^{2}$,

$$
\left(K u^{0}\right)(t)=S(t) u^{0}, \quad t \in\left[T_{1}, T_{2}\right], \quad u^{0} \in \mathcal{A}
$$

and $\delta=\frac{1-\eta}{2}$.

Lemma 6.4. If $f_{i} \in C^{1}(\mathbb{R}, \mathbb{R}), i \in \mathbb{Z}$, satisfy (3.2), (5.1) and (6.1), then for each $\varepsilon>0$ there exists an integer $k_{\varepsilon} \geq 0$ such that

$$
\sup _{|i| \geq k_{\varepsilon}}\left(f_{i}^{\prime}(0)-m_{i}\right) \leq \varepsilon
$$

In particular, if $\alpha_{0}$ is as in (4.2) then

$$
\sup _{|i| \geq k_{0}}\left(f_{i}^{\prime}(0)-m_{i}\right) \leq \frac{\alpha_{0}}{4} \text { for some integer } k_{0} \geq 0 .
$$

Proof. Define maps

$$
h_{i}(s)=f_{i}(s)-f_{i}^{\prime}(0) s, s \in \mathbb{R}, i \in \mathbb{Z} .
$$

Observe that $h_{i}$ are all of the class $C^{1}$, satisfy $h_{i}(0)=0$ for $i \in \mathbb{Z}$ and due to the mean value theorem, (3.2) and (6.7), we have for each $s \neq 0, i \in \mathbb{Z}$

$$
f_{i}^{\prime}(0)-m_{i} \leq \frac{\left|h_{i}(s)\right|}{|s|}+\frac{d_{i}}{|s|}=\left|h_{i}^{\prime}\left(\theta_{i} s\right)\right|+\frac{d_{i}}{|s|}=: \mathcal{R}_{i}(s),
$$

where $\theta_{i}$ denote some numbers from the interval $[0,1]$.

By assumption $d_{i} \geq 0, i \in \mathbb{Z}$, and $\left(d_{i}\right) \in \ell^{2}$, so we get

$$
d_{i} \rightarrow 0 \text { as }|i| \rightarrow \infty
$$

Writing now (6.8) with $s=\sqrt{d_{i}+\frac{1}{|i|}}, i \neq 0$, and using (6.1), (6.9) we obtain that $\mathcal{R}_{i}\left(\sqrt{d_{i}+\frac{1}{|i|}}\right) \rightarrow 0$ as $|i| \rightarrow \infty$, which leads to (6.5). 
Lemma 6.5. Under the assumptions of Theorem 6.2, that is (2.7), (3.1), (3.2), (4.2) and (6.1), there exist integers $k \in \mathbb{N}, N \geq 2$ and a number $T>0$ such that, given $\alpha_{0}$ as in (4.2) and $\mathcal{B}_{0}$ and $M$ as in Theorem 4.1, conditions (6.2) and (6.3) are satisfied for any $u^{0}, v^{0} \in \mathcal{B}_{0}$ with constants

$$
\begin{aligned}
D & =2\left(\max _{|i| \leq k}\left|f_{i}^{\prime}(0)-m_{i}\right|+\sup _{|i| \leq k} \sup _{|s| \leq M}\left|f_{i}^{\prime}(s)-f_{i}^{\prime}(0)\right|\right), \\
\kappa & =\left(\sqrt{2\left((4 v)^{2}+L_{M}^{2}\right)}+1\right) e^{L_{M} N T} \\
\eta & =e^{-\frac{\alpha_{0}}{2}(N-1) T} e^{L_{M} T}<1, \\
T_{1} & =T, T_{2}=N T .
\end{aligned}
$$

Proof. Observe that each component of any element of $\mathcal{B}_{0}$ belongs to $[-M, M]$. We remark that such interval exists because the space $\ell^{2}$ is embedded in $\ell^{\infty}$.

If $h_{i}$ is as in (6.7), then by (6.1), there exists $\delta \in(0, M)$ such that

$$
h_{i}^{\prime}(s) \leq \frac{1}{4} \alpha_{0} \text { whenever } i \in \mathbb{Z} \text { and }|s| \leq \delta .
$$

Setting for $u^{0}, v^{0} \in \mathcal{B}_{0}$

$$
u=S(t) u^{0}, \quad v=S(t) v^{0}, \quad U=u-v,
$$

we have from (1.2) that for each $t>0$

$$
U_{i}^{\prime}+(A U)_{i}=f_{i}\left(u_{i}\right)-f_{i}\left(v_{i}\right), i \in \mathbb{Z}
$$

We get by (2.7) and nonnegativity of $A$ that with some $\tilde{\theta}_{i} \in[0,1]$

$$
\frac{1}{2} \frac{\mathrm{d}}{\mathrm{d} t}\|U\|^{2} \leq \frac{1}{2} \frac{\mathrm{d}}{\mathrm{d} t}\|U\|^{2}+(A U, U)=\sum_{i \in \mathbb{Z}} f_{i}^{\prime}\left(\tilde{\theta}_{i} u_{i}+\left(1-\tilde{\theta}_{i}\right) v_{i}\right) U_{i}^{2} \leq L_{M}\|U\|^{2},
$$

which by the Gronwall inequality implies

$$
\|U(t)\| \leq e^{L_{M} t}\|U(0)\|, t \geq 0 .
$$

We also obtain from (1.2) that for each $t>0$

$$
U_{i}^{\prime}+(A U)_{i}=f_{i}^{\prime}(0) U_{i}+h_{i}\left(u_{i}\right)-h_{i}\left(v_{i}\right), i \in \mathbb{Z} .
$$

Then, using that $h_{i}\left(u_{i}\right)-h_{i}\left(v_{i}\right)=h_{i}^{\prime}\left(\theta_{i} u_{i}+\left(1-\theta_{i}\right) v_{i}\right) U_{i}$, where $\theta_{i} \in[0,1]$, we get

$$
\frac{1}{2} \frac{\mathrm{d}}{\mathrm{d} t}\|U\|^{2}+(A U, U)=\sum_{i \in \mathbb{Z}} f_{i}^{\prime}(0) U_{i}^{2}+\sum_{i \in \mathbb{Z}} h_{i}^{\prime}\left(\theta_{i} u_{i}+\left(1-\theta_{i}\right) v_{i}\right) U_{i}^{2}=: \mathcal{J}_{1}+\mathcal{J}_{2} .
$$


Concerning $\mathcal{J}_{1}$ we replace $f_{i}^{\prime}(0)$ by $f_{i}^{\prime}(0)-m_{i}+m_{i}$, and with the aid of (6.6), we estimate as follows

$$
\begin{aligned}
\mathcal{J}_{1} & =\sum_{|i| \leq k_{0}}\left(f_{i}^{\prime}(0)-m_{i}\right) U_{i}^{2}+\sum_{|i|>k_{0}}\left(f_{i}^{\prime}(0)-m_{i}\right) U_{i}^{2}+\sum_{i \in \mathbb{Z}} m_{i} U_{i}^{2} \\
& \leq \max _{|i| \leq k_{0}}\left|f_{i}^{\prime}(0)-m_{i}\right| \sum_{|i| \leq k_{0}} U_{i}^{2}+\frac{\alpha_{0}}{4}\|U\|^{2}+\sum_{i \in \mathbb{Z}} m_{i} U_{i}^{2} .
\end{aligned}
$$

To estimate $\mathcal{J}_{2}$ we consider $\delta$ as in (6.11) and apply Lemma 4.3 with $\varepsilon=\delta^{2}$ to obtain numbers $k_{\delta^{2}} \in \mathbb{N}$ and $T=T_{\delta^{2}}>0$ such that for

$$
k \geq \max \left\{k_{\delta^{2}}, k_{0}\right\}
$$

we have

$$
\sum_{|i|>k}\left|(S(t) w)_{i}\right|^{2}<\delta^{2} \text { for any } w \in \mathcal{B}_{0}, t \geq T .
$$

Using (6.15) we infer that

$$
\left|u_{i}\right| \leq \delta \text { and }\left|v_{i}\right| \leq \delta \text { for every }|i|>k \text { and } t \geq T .
$$

Hence, given any $|i|>k$ and $t \geq T$, we have $\left|\theta_{i} u_{i}+\left(1-\theta_{i}\right) v_{i}\right| \leq \delta$ and applying (6.11) we get

$$
h_{i}^{\prime}\left(\theta_{i} u_{i}+\left(1-\theta_{i}\right) v_{i}\right) \leq \frac{\alpha_{0}}{4} \text { for every }|i|>k \text { and } t \geq T .
$$

Consequently,

$$
\begin{aligned}
\mathcal{J}_{2} & =\sum_{|i| \leq k} h_{i}^{\prime}\left(\theta_{i} u_{i}+\left(1-\theta_{i}\right) v_{i}\right) U_{i}^{2}+\sum_{|i|>k} h_{i}^{\prime}\left(\theta_{i} u_{i}+\left(1-\theta_{i}\right) v_{i}\right) U_{i}^{2} \\
& \leq \sup _{|i| \leq k} \sup _{|s| \leq M}\left|h_{i}^{\prime}(s)\right| \sum_{|i| \leq k} U_{i}^{2}+\frac{\alpha_{0}}{4}\|U\|^{2}, t \geq T .
\end{aligned}
$$

The above estimates of $\mathcal{J}_{1}, \mathcal{J}_{2}$ together with (4.2) allow us to obtain from (6.13) a differential inequality of the form

$$
\begin{aligned}
\frac{1}{2} \frac{\mathrm{d}}{\mathrm{d} t}\|U\|^{2}+\frac{\alpha_{0}}{2}\|U\|^{2} & \leq \frac{1}{2} \frac{\mathrm{d}}{\mathrm{d} t}\|U\|^{2}+(A U, U)-(m U, U)-\frac{\alpha_{0}}{2}\|U\|^{2} \\
& \leq\left(\max _{|i| \leq k_{0}}\left|f_{i}^{\prime}(0)-m_{i}\right|+\sup _{|i| \leq k|s| \leq M} \sup _{|i|}\left|h_{i}^{\prime}(s)\right|\right) \\
& \sum_{|i| \leq k} U_{i}^{2}, t \geq T .
\end{aligned}
$$

Recalling (6.14) and letting $D=2\left(\max _{|i| \leq k}\left|f_{i}^{\prime}(0)-m_{i}\right|+\sup _{|i| \leq k} \sup _{|s| \leq M}\left|h_{i}^{\prime}(s)\right|\right)$, we now observe that $(6.16)$ yields

$$
\|U(t)\|^{2} \leq e^{-\alpha_{0}(t-T)}\|U(T)\|^{2}+D \sum_{|i| \leq k} \int_{T}^{t} U_{i}^{2}(s) \mathrm{d} s, t \geq T .
$$


Combining this with (6.12) gives

$$
\|U(t)\|^{2} \leq e^{-\alpha_{0}(t-T)} e^{2 L_{M} T}\|U(0)\|^{2}+D \sum_{|i| \leq k} \int_{T}^{t} U_{i}^{2}(s) \mathrm{d} s, t \geq T .
$$

Let $N \geq 2$ satisfy $e^{-\frac{\alpha_{0}}{2}(N-1) T} e^{L_{M} T}<1$. We also have by (2.8), (2.4) and (6.12)

$$
\begin{aligned}
\left\|U^{\prime}(t)\right\|^{2} & \leq 2\|A U(t)\|^{2}+2 \sum_{i \in \mathbb{Z}}\left|f_{i}\left(u_{i}(t)\right)-f_{i}\left(v_{i}(t)\right)\right|^{2} \\
& \leq 2\left((4 v)^{2}+L_{M}^{2}\right) e^{2 L_{M} t}\|U(0)\|^{2} .
\end{aligned}
$$

Hence we get (6.2) and (6.3) with constants as in the statement.

With minor changes in the argument, which are due to the fact that by (6.15) and invariance of $\mathcal{A}$ components of the solutions $u=S(t) u^{0}, v=S(t) v^{0}$ with $u^{0}, v^{0}$ from the attractor $\mathcal{A}$ satisfy $\left|u_{i}\right| \leq \delta$ and $\left|v_{i}\right| \leq \delta$ for every $|i|>k$ and $t \geq 0$, we have the following corollary.

Corollary 6.6. Under the assumptions of Theorem 6.2, there exists an integer $k>0$ such that, given $\alpha_{0}$ as in (4.2) and $M$ as in Theorem 4.1, conditions (6.2) and (6.3) are satisfied for any $u^{0}, v^{0} \in \mathcal{A}$ with constants

$$
\begin{aligned}
D & =2\left(\max _{|i| \leq k}\left|f_{i}^{\prime}(0)-m_{i}\right|+\sup _{|i| \leq k} \sup _{|s| \leq M}\left|f_{i}^{\prime}(s)-f_{i}^{\prime}(0)\right|\right), \\
\kappa & =\left(\sqrt{2\left((4 v)^{2}+L_{M}^{2}\right)}+1\right) e^{L_{M}}, \\
\eta & =e^{-\frac{\alpha_{0}}{2}} \\
T_{1} & =0, T_{2}=1 .
\end{aligned}
$$

Observe that the estimate of fractal dimension of the global attractor follows from (6.4) and (6.19) (or (6.10)). Theorem 6.2 is thus proved.

Corollary 6.7. Fractal dimension $\operatorname{dim}_{f}(\mathcal{A})$ is finite in $\ell^{p}$ for any $p \in[2, \infty]$ with the same estimate as given in (6.4), (6.19).

Proof. Indeed, we have $\|u\|_{p} \leq\|u\|_{\infty}^{1-\frac{2}{p}}\|u\|^{\frac{2}{p}}, u \in \ell^{2}$, and hence

$$
\|u\|_{p} \leq\|u\| \text { for } u \in \ell^{2}, p \in[2, \infty]
$$

Thus the claim follows by (6.2), (6.20) and invariance of $\mathcal{A}$ via the Lipschitz continuity

$$
\left\|S(1) u^{0}-S(1) v^{0}\right\|_{p} \leq \kappa\left\|u^{0}-v^{0}\right\|, u^{0}, v^{0} \in \mathcal{A}
$$

(see, e.g., [13, p. 95]). 
Corollary 6.8. Under the assumptions of Corollary 5.8, the global attractor $\mathcal{A}$ therein has finite fractal dimension in $\ell^{p}$ for any $p \in[2, \infty]$ with the estimate given in (6.4), where the constants $D, \eta, \kappa, T_{1}, T_{2}$ can be computed from (6.19) and $k \in \mathbb{N}$ from (6.11), (6.15), (6.6), (4.7), (4.3), provided that $m=\left(m_{i}\right) \in \ell^{\infty}$ and $\alpha_{0}>0$ satisfying (4.2) are given.

Considerations in the proof of Lemma 6.5 ensure that assumption (6.1) is a bit too strong and the result can be formulated as follows.

Corollary 6.9. Besides the assumptions of Theorem 4.4, that is (2.7), (3.1), (3.2) and (4.2), assume also (6.6) and (6.11). Under these assumptions, fractal dimension of the global attractor in Theorem 4.4 is finite in $\ell^{p}$ for any $p \in[2, \infty]$ and its estimate from above is given by (6.4) and (6.19).

Sometimes an $\ell^{\infty}$ sequence $m=\left(m_{i}\right)$, which moves the spectrum of $A$ to the right so that (4.2) holds, can be present in the equation. Then, instead of (6.7), a slightly different decomposition of nonlinearities is useful:

$$
f_{i}(s)=m_{i} s+h_{i}(s), \quad s \in \mathbb{R}, i \in \mathbb{Z},
$$

with $h_{i}$ satisfying (6.11). In this case we obtain the following result.

Corollary 6.10. Under the assumptions of Corollary 5.3 with $h_{i}$ satisfying (6.11), the global attractor $\mathcal{A}$ therein has finite fractal dimension in $\ell^{p}$ for any $p \in[2, \infty]$ and its estimate from above is given by (6.4) with constants $\kappa, \eta, T_{1}, T_{2}$ as in (6.19) and $D=2 \sup _{|i| \leq k} \sup _{|s| \leq M}\left|h_{i}^{\prime}(s)\right|$.

Proof. Following the proof of Lemma 6.5 note that in this case the left-hand side of (6.13) now is $(m U, U)+\sum_{i \in \mathbb{Z}} h_{i}^{\prime}\left(\theta_{i} u_{i}+\left(1-\theta_{i}\right) v_{i}\right) U_{i}^{2}$. Estimating then the quantity

$$
\mathcal{J}_{2}:=\sum_{i \in \mathbb{Z}} h_{i}^{\prime}\left(\theta_{i} u_{i}+\left(1-\theta_{i}\right) v_{i}\right) U_{i}^{2}
$$

as in the proof of Lemma 6.5 we will have the first line in (6.16) bounded from above by $\sup _{|i| \leq k} \sup _{|s| \leq M}\left|h_{i}^{\prime}(s)\right| \sum_{|i| \leq k} U_{i}^{2}$, with $k=k_{\delta^{2}}$ from Lemma 4.3, which implies (6.17) and (6.18) with constant $D$ equal now $2 \sup _{|i| \leq k} \sup _{|s| \leq M}\left|h_{i}^{\prime}(s)\right|$.

Remark 6.11. Observe that (6.11) is straightforward for the map $h$ defined in (5.4). Applying Corollary 6.10, we thus obtain an estimate of fractal dimension of the attractor from Example 5.4 in $\ell^{p}$ for any $p \in[2, \infty]$.

Remark 6.12. If besides (2.7) and (3.1) one assumes that (3.6) holds together with (4.2), then the semigroup $\{S(t): t \geq 0\}$ is a contraction for positive times, that is,

$$
\left\|S(t) u^{0}-S(t) v^{0}\right\| \leq e^{-\alpha_{0} t}\left\|u^{0}-v^{0}\right\|, \quad u^{0}, v^{0} \in \ell^{2}, t>0 .
$$

In such case, following [13, Corollary 3.1.18, Exercise 3.1.19], the attractor consists of a single point and thus has zero fractal dimension. 


\section{Exponential attractor}

We use below the Hausdorff semidistance $d(\cdot, \cdot)$ as in Theorem 4.4. Recalling that conditions (6.2) and (6.3) were verified in Lemma 6.5 for $u^{0}, v^{0}$ in the closed positively invariant set $\mathcal{B}_{0}$ from Theorem 4.1, we now use [13, Theorem. 3.2.1] to conclude the following result.

Theorem 7.1. Under the assumptions of Theorem 6.2, let $\mathcal{B}_{0}$ be the absorbing positively invariant set for the semigroup $\{S(t): t \geq 0\}$ in $\ell^{2}$ as in Theorem 4.1, and let $N, T, \eta$ be the constants from Lemma 6.5. Then there exists a compact set $\mathcal{E} \subset \mathcal{B}_{0}$ satisfying

$$
S(N T) \mathcal{E} \subset \mathcal{E}, \quad d\left(S(n N T) \mathcal{B}_{0}, \mathcal{E}\right) \leq r\left(\frac{1+\eta}{2}\right)^{n}, n \in \mathbb{N},
$$

for some $r>0$, and also such that its fractal dimension in $\ell^{2}$ is finite and estimated by

$$
\operatorname{dim}_{f}(\mathcal{E}) \leq \frac{\ln \mathfrak{m}_{\mathcal{B}_{\eta}}}{\ln \frac{2}{1+\eta}}
$$

$\mathfrak{m}_{\mathcal{B}_{\eta}}$ being the maximal number of $z^{j}$ in $\mathcal{B}_{\eta}:=\left\{z \in Z=C^{1}\left([T, N T] ; \ell^{2}\right):\|z\|_{Z} \leq\right.$ $\left.\frac{4 D^{\frac{1}{2}} \kappa}{1-\eta}\right\}$ with the property that $\int_{T}^{N T} \sum_{|i| \leq k}\left(z^{j}(t)-z^{l}(t)\right)_{i}^{2} d t>1$ for $j \neq l$.

Proof. Recalling that by Arzela-Ascoli theorem $n_{Z}(z)=\left(D \int_{T}^{N T} \sum_{|i| \leq k}(z(t))_{i}^{2} d t\right)^{\frac{1}{2}}$ is a compact seminorm on $Z=C^{1}\left([T, N T] ; \ell^{2}\right)$, and recalling Lemma 6.5, we get the result from Proposition B.4 specifying therein $\delta=\frac{1-\eta}{2}, X=\ell^{2}, \mathbb{M}=\mathcal{B}_{0}$, $V=S(N T)$ and $\left(K u^{0}\right)(t)=S(t) u^{0}$ for $t \in[T, N T], u^{0} \in \mathcal{B}_{0}$.

Following [13, Theorem 3.4.7], we now obtain the existence of an exponential attractor.

Theorem 7.2. Under the assumptions of Theorem 6.2, the semigroup $\{S(t): t \geq 0\}$ in $\ell^{2}$ has an exponential attractor, that is, there exists $\mathcal{M} \subset \ell^{2}$ such that

(i) $\mathcal{M}$ is compact in $\ell^{2}$,

(ii) $S(t) \mathcal{M} \subset \mathcal{M}$ for every $t \geq 0$,

(iii) $\mathcal{M}$ attracts exponentially bounded subsets of $\ell^{2}$ in the sense that, given any bounded set $\mathcal{B} \subset \ell^{2}$,

$$
d(S(t) \mathcal{B}, \mathcal{M}) \leq C_{\mathcal{B}} e^{-\omega t}, t \geq t_{\mathcal{B}}
$$

for some constants $C_{\mathcal{B}}>0, t_{\mathcal{B}}>0$ and $\omega>0(\omega$ independent of $\mathcal{B})$,

(iv) $\mathcal{M}$ has finite fractal dimension in $\ell^{2}$.

Moreover, fractal dimension of $\mathcal{M}$ in $\ell^{2}$ is estimated by

$$
\operatorname{dim}_{f}(\mathcal{M}) \leq 1+\frac{\ln \mathfrak{m}_{\mathcal{B}_{\eta}}}{\ln \frac{2}{1+\eta}}
$$


where $\mathfrak{m}_{\mathcal{B}_{\eta}}$ is as in Theorem 7.1. Also, $\mathcal{M}$ is contained in the set $\mathcal{B}_{0}$ from Theorem 4.1 and contains the global attractor $\mathcal{A}$ from Theorem 4.4 .

Proof. Recall from Theorem 4.1 and Lemma 6.5 that the $C^{0} \operatorname{semigroup~}\{S(t): t \geq 0\}$ on $\ell^{2}$ (see (3.5)) is both dissipative with positively invariant absorbing set $\mathcal{B}_{0}$, and quasi-stable on $\mathcal{B}_{0}$ at time $t^{*}=N T$ in the sense of [13, Definition 3.4.1]. Observe here that (6.2) and (6.3) hold for $u^{0}, v^{0} \in \mathcal{B}_{0}$ with $T_{1}=T$ and $T_{2}=N T$ as in Lemma 6.5.

Given $M$ as in Theorem 4.1 and denoting $u=S(\cdot) u^{0}$ observe also that for any $u^{0} \in \mathcal{B}_{0}$ we get from (2.4), (2.8)

$\left\|u^{\prime}(t)\right\| \leq\|A u(t)\|+\|F(u(t))-F(0)\|+\|F(0)\| \leq\left(4 v+L_{M}\right) M+\|g\|, t \geq 0$,

which ensures that

$$
\left\|S\left(t_{1}\right) u^{0}-S\left(t_{2}\right) u^{0}\right\| \leq\left(\left(4 v+L_{M}\right) M+\|g\|\right)\left|t_{1}-t_{2}\right|, t_{1}, t_{2} \in\left[0, t^{*}\right], u^{0} \in \mathcal{B}_{0} .
$$

Now we set $\mathcal{M}=\bigcup_{t \in\left[0, t^{*}\right]} S(t) \mathcal{E}$, where $t^{*}=N T$ and $\mathcal{E}$ comes from Theorem 7.1. If $\mathcal{B}$ is a bounded subset of $\ell^{2}$, then by Theorem 4.1 there exists $T_{\mathcal{B}} \geq 0$ such that $S\left(T_{\mathcal{B}}\right) \mathcal{B} \subset \mathcal{B}_{0}$. For $t \geq T_{\mathcal{B}}+t^{*}$, we have $t-T_{\mathcal{B}}=n_{t} t^{*}+r_{t}$ with $r_{t} \in\left[0, t^{*}\right], n_{t} \in \mathbb{N}$, and using that

$$
\left\|S(t) u^{0}-S(t) v^{0}\right\| \leq e^{L_{M} t^{*}}\left\|u^{0}-v^{0}\right\| \text { for } u^{0}, v^{0} \in \mathcal{B}_{0}, t \in\left[0, t^{*}\right]
$$

(see (6.12)), we get

$$
\begin{aligned}
& d(S(t) \mathcal{B}, \mathcal{M})=d\left(S\left(t-T_{\mathcal{B}}\right) S\left(T_{\mathcal{B}}\right) \mathcal{B}, \mathcal{M}\right) \leq d\left(S\left(t-T_{\mathcal{B}}\right) \mathcal{B}_{0}, \mathcal{M}\right) \\
& \quad \leq d\left(S\left(r_{t}\right) S\left(n_{t} t^{*}\right) \mathcal{B}_{0}, S\left(r_{t}\right) \mathcal{E}\right) \leq e^{L_{M} t^{*}} d\left(S\left(n_{t} t^{*}\right) \mathcal{B}_{0}, \mathcal{E}\right) \leq r e^{L_{M} t^{*}} e^{-\xi n_{t}} \\
& \quad \leq C_{\mathcal{B}} e^{-\frac{\xi}{t^{*} t}}
\end{aligned}
$$

where $\xi=-\ln \left(\frac{1+\eta}{2}\right)>0$ and $C_{\mathcal{B}}=r e^{L_{M} t^{*}+\xi+\frac{\xi T_{\mathcal{B}}}{t^{*}}}>0$. Hence we get the result.

Remark 7.3. There exists an exponential attractor for lattice systems in Corollary 5.3 with $h_{i}$ satisfying (6.11), and for systems in Corollary 5.8; in particular for (5.5) in Example 5.4.

\section{Acknowledgements}

The authors thank Stefanie Sonner for suggesting a study of existence of exponential attractors for lattice dynamical systems under mild assumptions. The authors also thank the anonymous referee for the positive comments and helpful suggestions which improved the final version of the paper. 
Open Access. This article is distributed under the terms of the Creative Commons Attribution 4.0 International License (http://creativecommons.org/licenses/by/4.0/), which permits unrestricted use, distribution, and reproduction in any medium, provided you give appropriate credit to the original author(s) and the source, provide a link to the Creative Commons license, and indicate if changes were made.

Publisher's Note Springer Nature remains neutral with regard to jurisdictional claims in published maps and institutional affiliations.

\section{Appendix A: Discretized Schrödinger operator}

In this section, we obtain a discretized version of the well-known Arendt and Batty's result reported in [4, Theorem 1.2]. To this purpose, we define a suitable family $\mathcal{G}$ of subsets of $\mathbb{Z}$ being a counterpart of a family of sets in [4] which contain arbitrarily large balls.

Definition A.1. We say that $G \subset \mathbb{Z}$ belongs to $\mathcal{G}$ if and only if for any $n \in \mathbb{N}$ there exists $j_{n} \in \mathbb{Z}$ such that $\left\{i \in \mathbb{Z}:\left|i-j_{n}\right|<n\right\} \subset G$.

Consider the bounded linear operator $A \in \mathcal{L}\left(\ell^{p}\right), p \in[1, \infty]$, defined in (2.1). The linear semigroup $e^{-A t}$ generated by $-A$ on $\ell^{p}$ can be expressed as the convolution with the discrete Gaussian kernel (see [25, Theorems 3.21, 3.28]).

Proposition A.2. The linear semigroup $e^{-A t}$ on $\ell^{p}$ with $p \in[1, \infty]$ is given by

$$
\left(e^{-A t} u^{0}\right)_{i}=\sum_{j \in \mathbb{Z}} e^{-2 v t} I_{i-j}(2 v t) u_{j}^{0}, t \geq 0, u^{0} \in \ell^{p}, i \in \mathbb{Z},
$$

where $I_{n}:[0, \infty) \rightarrow[0, \infty), n \in \mathbb{Z}$, defined by

$$
I_{n}(x)=I_{-n}(x)=\sum_{m=0}^{\infty} \frac{1}{m !(m+n) !}\left(\frac{x}{2}\right)^{2 m+n}, \quad x \geq 0, \quad n \in \mathbb{N}_{0},
$$

are the modified Bessel functions.

Proof. We have $I_{n}(0)=0, n \neq 0, I_{0}(0)=1$, and

$$
I_{n}^{\prime}(x)=\frac{1}{2}\left(I_{n-1}(x)+I_{n+1}(x)\right), n \in \mathbb{Z}, x \geq 0 .
$$

We denote the right-hand side of (A.1) by $u_{i}(t)$. By evaluating it at $t=0$ and computing its derivative we obtain

$$
\left\{\begin{array}{l}
u_{i}^{\prime}=v\left[u_{i+1}+u_{i-1}-2 u_{i}\right], \quad t>0, \quad i \in \mathbb{Z}, \\
u_{i}(0)=u_{i}^{0}, \quad i \in \mathbb{Z}
\end{array}\right.
$$

which yields the claim.

Now we are ready to give equivalent conditions for the discrete Schrödinger operator $-A-c$ with $0 \leq c \in \ell^{\infty}$ to generate a uniformly exponentially stable semigroup. 
Theorem A.3. Let $0 \leq c \in \ell^{\infty}$ and let the operator $A \in \mathcal{L}\left(\ell^{p}\right), p \in[1, \infty]$, be given as in (2.1). The following conditions are equivalent:

(i) $\left\|e^{(-A-c) t}\right\|_{\mathcal{L}\left(\ell^{p}\right)}<1$ for all $t>0, p \in[1, \infty]$,

(ii) $\left\|e^{(-A-c) t_{0}}\right\|_{\mathcal{L}\left(\ell^{2}\right)}<1$ for some $t_{0}>0$,

(iii) $s_{p}(-A-c)=\sup \left\{\operatorname{Re} \lambda: \lambda \in \sigma_{p}(-A-c)\right\}<0$, that is, $\operatorname{Re} \lambda<0$ for $\lambda \in$ $\sigma_{p}(-A-c)$ for all $p \in[1, \infty]$,

(iv) there exists $\alpha_{0}>0$ such that $((A+c) u, u) \geq \alpha_{0}\|u\|^{2}, u \in \ell^{2}$,

(v) $\sum_{i \in G} c_{i}=\infty$ for any set $G$ from a family $\mathcal{G}$ in Definition A.1.

(vi) there exist $C>0, N \in \mathbb{N}$ such that $\sum_{i=j-N}^{j+N} c_{i} \geq C$ for all $j \in \mathbb{Z}$.

Proof. (i) $\Rightarrow$ (iii). Note that by [18, Corollary IV.3.12] the spectral bound

$$
s_{p}(-A-c):=\sup \left\{\operatorname{Re} \lambda: \lambda \in \sigma_{p}(A-c)\right\}
$$

is equal to the growth bound

$$
\begin{aligned}
& \omega_{0, p}(-A-c):=\inf \left\{a \in \mathbb{R}:\left\|e^{(-A-c) t}\right\|_{\mathcal{L}\left(\ell^{p}\right)} \leq M_{a} e^{a t} \text { for some } M_{a}\right. \\
& \geq 1 \text { and all } t \geq 0\} .
\end{aligned}
$$

Hence using the spectral radius, we get (see [18, Proposition IV.2.2])

$$
e^{\omega_{0, p}(-A-c) t}=r_{p}\left(e^{(-A-c) t}\right) \leq\left\|e^{(-A-c) t}\right\|_{\mathcal{L}\left(\ell^{p}\right)}, t \geq 0 .
$$

Thus if $\left\|e^{(-A-c) t_{0}}\right\|_{\mathcal{L}\left(\ell^{p}\right)}<1$ for some $t_{0}>0$, then $\omega_{0, p}(-A-c)=s_{p}(-A-c)<0$.

(iii) $\Rightarrow$ (iv). In the Hilbert case $p=2$ the operator $-A-c$ is self-adjoint on $\ell^{2}$, hence we have $\sigma_{2}(-A-c) \subset \mathbb{R}$ and

$$
\begin{aligned}
& s_{2}(-A-c)=\sup \left\{\lambda: \lambda \in \sigma_{2}(-A-c)\right\} \\
& \quad=\sup \{((-A-c) u, u):\|u\|=1\}=-\inf \{((A+c) u, u):\|u\|=1\} .
\end{aligned}
$$

If $s_{2}(-A-c)<0$ then

$$
\alpha_{0}:=\inf \{((A+c) u, u):\|u\|=1\}>0
$$

and we obtain (iv).

(iv) $\Rightarrow$ (v). Using $B$ as in (2.3), we have for $u \in \ell^{2}$ with $\|u\|=1$

$$
0<\alpha_{0} \leq\left(B^{*} B u, u\right)+(c u, u)=\sum_{i \in \mathbb{Z}}(B u)_{i}^{2}+\sum_{i \in \mathbb{Z}} c_{i} u_{i}^{2} .
$$

Let $G \in \mathcal{G}$ and observe that we can find $u^{n} \in \ell^{2}$ such that $\left\|u^{n}\right\|=1$ and $u_{i}^{n}=0$ for $i \notin G$, whereas

$$
\left\|B u^{n}\right\| \rightarrow 0 \text { and }\left\|u^{n}\right\|_{\infty} \rightarrow 0 \text { as } n \rightarrow \infty
$$


For example, if $j_{n} \in \mathbb{Z}, n \in \mathbb{N}$, is such that $\left\{i \in \mathbb{Z}:\left|i-j_{n}\right|<n\right\} \subset G$, then we can use

$$
u_{i}^{n}=\left\{\begin{array}{ll}
\frac{1}{\sqrt{2(n-1)}}, & \left|i-j_{n}\right|<n, i \neq j_{n} \\
0, & \left|i-j_{n}\right| \geq n \text { or } i=j_{n}
\end{array}, n \geq 2 .\right.
$$

In this case, we know that $\left\|u^{n}\right\|_{\infty}=\frac{1}{\sqrt{2(n-1)}},\left\|u^{n}\right\|=1,\left\|B u^{n}\right\|=0$. We will have

$$
0<\alpha_{0} \leq\left\|B u_{n}\right\|^{2}+\left\|u^{n}\right\|_{\infty}^{2} \sum_{i \in G} c_{i},
$$

which implies that $\sum_{i \in G} c_{i}=\infty$.

(v) $\Rightarrow$ (vi). Suppose contrary to the claim that there exists a sequence $j_{n} \in \mathbb{Z}, n \in \mathbb{N}$, such that

$$
\sum_{i=j_{n}-n}^{j_{n}+n} c_{i}<\frac{1}{2^{n}}
$$

Define

$$
G_{m}=\bigcup_{n>m}\left\{i \in \mathbb{Z}:\left|i-j_{n}\right| \leq n\right\}, m \in \mathbb{N},
$$

and note that $G_{m} \in \mathcal{G}$, since for $n>m$ we have $\left\{i \in \mathbb{Z}:\left|i-j_{n}\right| \leq n\right\} \subset G_{m}$ and for $1 \leq n \leq m$ we have

$$
\left\{i \in \mathbb{Z}:\left|i-j_{m+1}\right| \leq n\right\} \subset\left\{i \in \mathbb{Z}:\left|i-j_{m+1}\right| \leq m+1\right\} \subset G_{m}
$$

Hence we obtain

$$
\infty=\sum_{i \in G_{m}} c_{i} \leq \sum_{n=m+1}^{\infty} \sum_{i=j_{n}-n}^{j_{n}+n} c_{i} \leq \sum_{n=m+1}^{\infty} \frac{1}{2^{n}}=\frac{1}{2^{m}}, m \in \mathbb{N},
$$

which is absurd.

(vi) $\Rightarrow$ (i). Let $t>0$ and assume that $\|c\|_{\infty}<\frac{1}{t}$. This can be done since otherwise we could consider $\lambda c$ instead of $c$ with $\lambda=\frac{1}{2} \min \left\{\frac{1}{t\|c\|_{\infty}}, 1\right\}$ having by [9, Proposition 10.22] for any $p \in[1, \infty]$

$$
\left\|e^{(-A-c) t}\right\|_{\mathcal{L}\left(\ell^{p}\right)} \leq\left\|e^{(-A-\lambda c) t}\right\|_{\mathcal{L}\left(\ell^{p}\right)}, t \geq 0,
$$

because the semigroups $\left\{e^{(-A-c) t}: t \geq 0\right\}$ and $\left\{e^{(-A-\lambda c) t}: t \geq 0\right\}$ on $\ell^{p}$ are positive (see [3, Example C-II.1.13 a)]) and for $u^{0} \geq 0, u^{0} \in \ell^{p}$ $e^{(-A-\lambda c) t} u^{0}=e^{(-A-c) t} u^{0}+\int_{0}^{t} e^{(-A-c)(t-s)}(1-\lambda) c e^{(-A-\lambda c) s} u^{0} \mathrm{~d} s \geq e^{(-A-c) t} u^{0}$. 
Since for the operator $A$ considered on $\ell^{\infty}$ we have $A \mathbb{1}=0$, it follows that $e^{-A t} \mathbb{1}=\mathbb{1}$ and

$$
e^{(-A-c) t} \mathbb{1}=\mathbb{1}-\int_{0}^{t} e^{-A(t-s)} c e^{(-A-c) s} \mathbb{1} \mathrm{d} s
$$

Hence we get

$$
e^{(-A-c) t} \mathbb{1}=\mathbb{1}-\int_{0}^{t} e^{-A(t-s)} c \mathrm{~d} s+\int_{0}^{t} e^{-A(t-s)} c \int_{0}^{s} e^{-A(s-r)} c e^{(-A-c) r} \mathbb{1} \mathrm{d} r \mathrm{~d} s .
$$

Moreover, due to positivity of $\left\{e^{(-A-c) t}: t \geq 0\right\}$ and $\left\{e^{-A t}: t \geq 0\right\}$ on $\ell^{\infty}$ (see again [3, Example C-II.1.13 a)]), we know that

$$
e^{(-A-c) t} u=e^{-A t} u^{0}-\int_{0}^{t} e^{-A(t-s)} c e^{(-A-c) s} u^{0} \mathrm{~d} s \leq e^{-A t} u^{0}, u^{0} \geq 0 .
$$

Thus we obtain $e^{(-A-c) t} \mathbb{1} \leq e^{-A t} \mathbb{1}=\mathbb{1}$. Since $c \leq\|c\|_{\infty} \mathbb{1}$, we get

$$
\begin{aligned}
& \int_{0}^{t} e^{-A(t-s)} c \int_{0}^{s} e^{-A(s-r)} c e^{(-A-c) r} \mathbb{1} \mathrm{d} r \mathrm{~d} s \\
& \quad \leq\|c\|_{\infty} \int_{0}^{t} e^{-A(t-s)} c \int_{0}^{s} e^{-A(s-r)} \mathbb{1} \mathrm{d} r \mathrm{~d} s=\|c\|_{\infty} \int_{0}^{t} s e^{-A(t-s)} c \mathrm{~d} s \\
& \quad=\|c\|_{\infty} \int_{0}^{t}(t-s) e^{-A s} c \mathrm{~d} s \leq\|c\|_{\infty} t \int_{0}^{t} e^{-A s} c \mathrm{~d} s
\end{aligned}
$$

Hence we obtain

$$
e^{(-A-c) t} \mathbb{1} \leq \mathbb{1}-\left(1-t\|c\|_{\infty}\right) \int_{0}^{t} e^{-A s} c \mathrm{~d} s
$$

By assumption there exist $C>0, N \in \mathbb{N}$ such that $\sum_{j=i-N}^{i+N} c_{j} \geq C$ for all $i \in \mathbb{Z}$. Then, given $i \in \mathbb{Z}$, we have by Proposition A.2

$$
\begin{aligned}
& \left(e^{-A s} c\right)_{i}=\sum_{j \in \mathbb{Z}} e^{-2 v s} I_{i-j}(2 v s) c_{j} \geq \sum_{j=i-N}^{i+N} e^{-2 v s} I_{i-j}(2 v s) c_{j} \\
& \geq e^{-2 v s} \min _{|l| \leq N} I_{l}(2 v s) \sum_{j=i-N}^{i+N} c_{j} \geq C e^{-2 v s} \min _{0 \leq l \leq N} I_{l}(2 v s) \geq C e^{-2 v s} I_{N}(2 v s), s \geq 0,
\end{aligned}
$$

where the last inequality follows from

$$
I_{n}(x)>\left(1+\frac{n}{x}\right) I_{n+1}(x) \geq I_{n+1}(x), \quad x>0, \quad n \in \mathbb{N}_{0}
$$

(see, e.g., [26, Proposition 1]). Hence, letting $\varepsilon(t)=\int_{0}^{t} C e^{-2 v s} I_{N}(2 v s) d s>0$, we obtain

$$
\int_{0}^{t}\left(e^{-A s} c\right)_{i} \mathrm{~d} s \geq \varepsilon(t) \text { for all } i \in \mathbb{Z}
$$


It follows that

$$
\left(e^{(-A-c) t} \mathbb{1}\right)_{i} \leq 1-\left(1-t\|c\|_{\infty}\right) \varepsilon(t), i \in \mathbb{Z},
$$

and so using the adjoint semigroup and [9, Proposition 10.22] we get

$$
\left\|e^{(-A-c) t}\right\|_{\mathcal{L}\left(\ell^{1}\right)}=\left\|e^{(-A-c) t}\right\|_{\mathcal{L}\left(\ell^{\infty}\right)}=\left\|e^{(-A-c) t} \mathbb{1}\right\|_{\infty}<1 .
$$

Furthermore, from the Riesz-Thorin theorem, we obtain

$$
\left\|e^{(-A-c) t}\right\|_{\mathcal{L}\left(\ell^{p}\right)}<1, \quad p \in[1, \infty]
$$

which yields the claim.

(i) $\Leftrightarrow$ (ii). If (ii) holds, the above considerations imply that (iii) holds for $p=2$, which in turn yields (iv) and (i). The converse implication is straightforward.

\section{Appendix B: Abstract estimate of fractal dimension}

The estimate of the fractal dimension of invariant set in $\ell^{2}$ used in this article comes from the monograph by Chueshov [13, Theorem 3.1.21] (see also [14, Theorem 2.14]), which we recall below for the completeness of the presentation.

Proposition B.1. Let $X$ be a Banach space and $\mathbb{M}$ be a bounded closed set in $X$. Assume that there exists a mapping $V: \mathbb{M} \rightarrow X$ satisfying $\mathbb{M} \subset V \mathbb{M}$ and there is an auxiliary normed space $Z$ and a mapping $K: \mathbb{M} \rightarrow Z$ such that for some constants $\eta \in[0,1)$ and $\kappa>0$ we have

$$
\begin{aligned}
& \|K x-K y\|_{Z} \leq \kappa\|x-y\|_{X}, x, y \in \mathbb{M}, \\
& \|V x-V y\|_{X} \leq \eta\|x-y\|_{X}+n_{Z}(K x-K y), x, y \in \mathbb{M},
\end{aligned}
$$

where $n_{Z}$ is some compact seminorm on Z, i.e., $n_{Z}$ satisfies

$$
n_{Z}(x+y) \leq n_{Z}(x)+n_{Z}(y), \quad n_{Z}(\lambda x)=|\lambda| n_{Z}(x), x, y \in Z, \lambda \in \mathbb{R}
$$

and for any bounded sequence $z_{n} \in Z$ there exists a Cauchy subsequence $z_{n_{j}}$ with respect to $n_{Z}$, that is, $n_{Z}\left(z_{n_{j}}-z_{n_{l}}\right) \rightarrow 0$ as $j, l \rightarrow \infty$.

Then $\mathbb{M}$ is compact in $X$, its fractal dimension $\operatorname{dim}_{f}^{X}(\mathbb{M})$ in $X$ is finite and, given any $\delta \in(0,1-\eta)$,

$$
\operatorname{dim}_{f}^{X}(\mathbb{M}) \leq \frac{\ln \mathfrak{m}_{Z}\left(2 \kappa \delta^{-1}\right)}{\ln \frac{1}{\eta+\delta}}
$$

where $\mathfrak{m}_{Z}\left(2 \kappa \delta^{-1}\right)$ is the maximal number of points $z_{j}$ in the ball $\left\{z \in Z:\|z\|_{Z} \leq\right.$ $\left.2 \kappa \delta^{-1}\right\}$ possessing the property that $n_{Z}\left(z_{j}-z_{l}\right)>1$ whenever $j \neq l$.

Instead of Proposition B.1, one can use here the following corollary. 
Corollary B.2. Let $X$ be a Banach space and let $\mathbb{M} \neq \varnothing$ be a bounded and closed subset of $X$ and let a map $V: \mathbb{M} \rightarrow X$ be such that $\mathbb{M} \subset V \mathbb{M}$. Assume that $Y, Z$ are normed spaces, $P: Z \rightarrow Y$ is a linear compact operator and there exists a map $K: \mathbb{M} \rightarrow Z$ satisfying for some $\eta \in[0,1)$ and $\mu, \kappa>0$

$$
\begin{aligned}
& \|V x-V y\|_{X} \leq \eta\|x-y\|_{X}+\mu\|P(K x-K y)\|_{Y}, x, y \in \mathbb{M}, \\
& \|K x-K y\|_{Z} \leq \kappa\|x-y\|_{X}, x, y \in \mathbb{M} .
\end{aligned}
$$

Then $\mathbb{M}$ is a compact set in $X$ and for any $\delta \in(0,1-\eta)$

$$
\operatorname{dim}_{f}^{X}(\mathbb{M}) \leq \frac{\ln \mathfrak{m}_{Z}\left(2 \mu \kappa \delta^{-1}\right)}{\ln \frac{1}{\eta+\delta}}
$$

where $\mathfrak{m}_{Z}\left(2 \mu \kappa \delta^{-1}\right)$ is the maximal number of points $z_{j}$ in the ball $\left\{z \in Z:\|z\|_{Z} \leq\right.$ $\left.2 \mu \kappa \delta^{-1}\right\}$ possessing the property that $\left\|P\left(z_{j}-z_{l}\right)\right\|_{Y}>1$ whenever $j \neq l$.

Proof. The result follows from Proposition B.1, since $n_{Z}(z):=\mu\|P z\|_{Y}, z \in Z$, is a compact seminorm on $Z$.

Remark B.3. Concerning finite fractal dimension of $\mathbb{M}$ under the assumptions as in Corollary B.2, we remark that it was earlier reported by Czaja and Efendiev in [15, Theorem 2.1] with $P$ being a compact embedding of $Z$ into $Y$ and $\mathbb{M}$ being invariant under $V$. The authors therein used Kolmogorov $\varepsilon$-entropy (see [2]) and for $\eta \in\left[0, \frac{1}{2}\right.$ ) obtained with any $\theta \in\left(0, \frac{1}{2}-\eta\right)$ the estimate of the form

$$
\operatorname{dim}_{f}^{X}(\mathbb{M}) \leq \frac{\ln N_{\frac{\theta}{\mu \kappa}}^{Y}\left(\mathcal{B}_{1}\right)}{\ln \frac{1}{2(\eta+\theta)}},
$$

where $N_{\frac{\theta}{\mu \kappa}}^{Y}\left(\mathcal{B}_{1}\right)$ denotes the minimal number of closed balls in $Y$ of radius $\frac{\theta}{\mu \kappa}$ necessary to cover the closed unit ball $\mathcal{B}_{1}$ in $Z$. In this case, one can apply Corollary B.2 with $2 \eta$ in the role of $\eta$ and $\delta=2 \theta$ making the denominators in (7.3) and (7.4) equal $\ln \frac{1}{2(\eta+\theta)}$. Observe that the quantity $\mathfrak{m}_{Z}\left(2 \mu \kappa \delta^{-1}\right)$ appearing in (7.3) can be viewed as the maximal number of points $z_{j}$ in the closed unit ball in $Z$ having the property that $\left\|z_{j}-z_{l}\right\|_{Y}>\frac{\theta}{\mu \kappa}$ for $j \neq l$. Then, given such points, there exists a cover of the closed unit ball $\mathcal{B}_{1}$ in $Z$ by $\mathfrak{m}_{Z}\left(\mu \kappa \theta^{-1}\right)$ closed balls in $Y$ of radius $\frac{\theta}{\mu \kappa}$.

Concerning estimate of fractal dimension involving discrete exponential attractor, we finally recall the following result by Chueshov [13, Theorem 3.2.1].

Proposition B.4. Let $X$ be a Banach space and $\mathbb{M}$ be a bounded closed set in $X$. Assume that there is a map $V: \mathbb{M} \rightarrow \mathbb{M}$ and there also exists a map $K$ from $\mathbb{M}$ into an auxiliary normed space $Z$ such that (B.1) and (B.2) hold with some constants $\eta \in$ $[0,1), \kappa>0$, and with $n_{Z}$ being a compact seminorm on $Z$. Then, given $\delta \in(0,1-\eta)$, there exists a compact set $\mathbb{A}_{\delta} \subset \mathbb{M}$ satisfying

$$
V \mathbb{A}_{\delta} \subset \mathbb{A}_{\delta}, \quad d\left(V^{n} \mathbb{M}, \mathbb{A}_{\delta}\right) \leq r(\eta+\delta)^{n}, n \in \mathbb{N},
$$


for some $r>0$, and also such that its fractal dimension in $X$ is finite and estimated by

$$
\operatorname{dim}_{f}^{X}\left(\mathbb{A}_{\delta}\right) \leq \frac{\ln \mathfrak{m}_{Z}\left(2 \kappa \delta^{-1}\right)}{\ln \frac{1}{\eta+\delta}},
$$

$\mathfrak{m}_{Z}\left(2 \kappa \delta^{-1}\right)$ being the maximal number of $z_{j}$ in $\left\{z \in Z:\|z\|_{Z} \leq 2 \kappa \delta^{-1}\right\}$ having the property that $n_{Z}\left(z_{j}-z_{l}\right)>1$ for $j \neq l$.

\section{REFERENCES}

[1] A.Y. Abdallah, Exponential attractors for first-order lattice dynamical systems, J. Math. Anal. Appl. 339 (2008), 217-224.

[2] M. Anguiano, A. Haraux, The $\varepsilon$-entropy of some infinite dimensional compact ellipsoids and fractal dimension of attractors, Evol. Equ. Control Theory 6 (2017), 345-356.

[3] W. Arendt, Characterization of positive semigroups on Banach lattices. In One-Parameter Semigroups of Positive Operators R. Nagel, Springer, 1986, 247-291.

[4] W. Arendt, C. Batty, Exponential stability of a diffusion equation with absorption, Differ. Int. Equ. 6 (1993), 1009-1024.

[5] J.M. Arrieta, J.W. Cholewa, T. Dlotko, A. Rodríguez-Bernal, Asymptotic behavior and attractors for reaction diffusion equations in unbounded domains, Nonlinear Anal. 56 (2004), 515-554.

[6] J.M. Arrieta, N. Moya, A. Rodríguez-Bernal, On the finite dimension of attractors of parabolic problems in $\mathbb{R}^{N}$ with general potentials, Nonlinear Anal. 68 (2008), 1082-1099.

[7] A.V. Babin, M.I. Vishik, Attractors of partial differential evolution equations in unbounded domains, Proc. R. Soc. Edinburgh Sect. A 116 (1990), 221-243.

[8] P.W. Bates, K. Lu, B. Wang, Attractors for lattice dynamical systems, Int. J. Bifur. Chaos Appl. Sci. Eng. 11 (2001), 143-153.

[9] A. Bátkai, M. Kramar Fijavž, A. Rhandi, Positive Operator Semigroups-From Finite to Infinite Dimensions, Birkhäuser, 2017.

[10] T. Caraballo, F. Morillas, J. Valero, Asymptotic behaviour of a logistic lattice system, Discrete Contin. Dyn. Syst. 34 (2014), 4019-4037.

[11] J.W. Cholewa, A. Rodríguez-Bernal, Dissipative mechanism of a semilinear higher order parabolic equation in $\mathbb{R}^{N}$, Nonlinear Anal. 75 (2012), 3510-3530.

[12] S.-N. Chow, J. Mallet-Paret, Pattern formation and spatial chaos in lattice dynamical systems-Part I, IEEE Trans. Circuits Syst. I Fund. Theory Appl. 42 (1995), 746-751.

[13] I. Chueshov, Dynamics of Quasi-Stable Dissipative Systems, Springer, 2015.

[14] I. Chueshov, I. Lasiecka, Long-Time Behavior of Second Order Evolution Equations with Nonlinear Damping, Memoirs of the American Mathematical Society 195 (912), AMS, 2008.

[15] R. Czaja, M. Efendiev, A note on attractors with finite fractal dimension, Bull. Lond. Math. Soc. 40 (2008), 651-658.

[16] M. Efendiev, A. Miranville, S. Zelik, Global and exponential attractors for nonlinear reactiondiffusion systems in unbounded domains, Proc. R. Soc. Edinburgh Sect. A 134 (2004), 271-315.

[17] M. Efendiev, S. Zelik, The attractor for a nonlinear reaction-diffusion system in an unbounded domain, Commun. Pure Appl. Math. 54 (2001), 625-688.

[18] K.-J. Engel, R. Nagel, One-Parameter Semigroups for Linear Evolution Equations, Springer, Berlin, 2000.

[19] X. Fan, Exponential attractor for a first-order dissipative lattice dynamical system, J. Appl. Math. (2008), ID 354652, 8 pages.

[20] J.K. Hale, Asymptotic Behavior of Dissipative Systems, American Mathematical Society, Providence, 1988.

[21] X. Han, Exponential attractors for lattice dynamical systems in weighted spaces, Discrete Contin. Dyn. Syst. 31 (2011), 445-467.

[22] O.A. Ladyženskaya, Attractors for Semigroups and Evolution Equations, Cambridge University Press, Cambridge, 1991. 
[23] X. Li, K. Wei, H. Zhang, Exponential attractors for lattice dynamical systems in weighted spaces, Acta Appl. Math. 114 (2011), 157-172.

[24] X. Li, C. Zhong, Attractors for partly dissipative lattice dynamic systems in $\ell^{2} \times \ell^{2}, J$. Comput. Appl. Math. 177 (2005), 159-174.

[25] T. Lindeberg, Scale-Space Theory in Computer Vision, Springer, Berlin, 1994.

[26] I. Nåsell, Inequalities for modified Bessel functions, Math. Comput. 28 (1974), 253-256.

[27] A. Rodríguez-Bernal, B. Wang, Attractors for partly dissipative reaction diffusion systems in $\mathbb{R}^{n}$, J. Math. Anal. Appl. 252 (2000), 790-803.

[28] A. Slavík, Invariant regions for systems of lattice reaction-diffusion equations, J. Differ. Equ. 263 (2017), 7601-7626.

[29] R. Temam, Infinite-Dimensional Dynamical Systems in Mechanics and Physics, Second Edition, Springer, Berlin, 1997.

[30] B. Wang, Attractors for reaction-diffusion equations in unbounded domains, Phys. D 128 (1999), $41-52$.

[31] B. Wang, Dynamics of systems on infinite lattices, J. Differ. Equ. 221 (2006), 224-245.

[32] B. Wang, Asymptotic behavior of non-autonomous lattice systems, J. Math. Anal. Appl. 331 (2007), 121-136.

[33] S. Zhou, Attractors for first order dissipative lattice dynamical systems, Phys. D 178 (2003), 51-61.

[34] S. Zhou, Attractors and approximations for lattice dynamical systems, J. Differ. Equ. 200 (2004), 342-368.

[35] S. Zhou, W. Shi, Attractors and dimension of dissipative lattice systems, J. Differ. Equ. 224 (2006), 172-204.

[36] B. Zinner, Existence of traveling wavefront solutions for the discrete Nagumo equation, J. Differ. Equ. 96 (1992), 1-27.

Jan W. Cholewa and Radosław Czaja Institute of Mathematics

University of Silesia in Katowice

Bankowa 14

40-007 Katowice

Poland

E-mail: jan.cholewa@us.edu.pl

Radostaw Czaja

E-mail: radoslaw.czaja@us.edu.pl 